Research Article

\title{
Fractional Cauchy Problem with Riemann-Liouville Fractional Delta Derivative on Time Scales
}

\author{
Jiang Zhu and Ying Zhu \\ School of Mathematics and Statistics, Jiangsu Normal University, Xuzhou 221116, China \\ Correspondence should be addressed to Jiang Zhu; jzhuccy@163.com
}

Received 9 August 2013; Accepted 6 October 2013

Academic Editor: Ali H. Bhrawy

Copyright (C) 2013 J. Zhu and Y. Zhu. This is an open access article distributed under the Creative Commons Attribution License, which permits unrestricted use, distribution, and reproduction in any medium, provided the original work is properly cited.

The $\Delta$-power function and fractional $\Delta$-integrals and fractional $\Delta$-differential are defined, and then the definitions and properties of $\Delta$-Mittag-Leffler function are given. The properties of fractional $\Delta$-integrals and fractional $\Delta$-differential on time scales are discussed in detail. After that, the existence of the solution and the dependency of the solution upon the initial value for Cauchy type problem with fractional $\Delta$-derivative are studied. Also the explicit solutions to homogeneous fractional $\Delta$-differential equations and nonhomogeneous fractional $\Delta$-differential equations are derived by using Laplace transform method.

\section{Introduction}

The fractional differential equation theory is an important subject of mathematics, which includes continuous fractional differential equations and discrete fractional difference equations. The theory of fractional differential equations has gained considerable popularity and importance during the past three decades or so. Many applications in numerous seemingly diverse and widespread fields of science and engineering have been gained. It does indeed provide several potentially useful tools for solving differential and integral equations and various other problems involving special functions of mathematical physics as well as their extensions and generalizations in one and more variables. About these advances, one can refer to $[1,2]$, the books $[3,4]$, and the references of them. For the recent developments about continuous fractional differential equations and discrete fractional difference equations, one can refer to [5-11]. To unify differential equations and difference equations, Higer proposed firstly the time scale and built the relevant basic theories (see [12-15]). Recently, some authors studied fractional calculus on time scales (see $[16,17]$ ), where Williams [16] gives a definition of fractional $\nabla$-integral and $\nabla$ derivative on time scales to unify three cases of specific time scales. Bastos gives definitions of fractional $\Delta$-integral and
$\Delta$-derivative on time scales by the inverse of Laplace transform in [17].

Inspired by these works, the aim of this paper is to give a new definition of fractional $\Delta$-integral and $\Delta$-derivative on general time scales and then study some fractional differential equations on time scales. To define the fractional $\Delta$-integral and fractional $\Delta$-derivative, we would need to obtain a definition of fractional order power functions on time scales to generalize the monomials. Different from definition of $\nabla$ power functions by axiomatization method in [16], we define fractional $\Delta$-power functions on general time scales by using inversion of time scale Laplace transform and shift transform in Section 3, and Riemann-Liouville fractional $\Delta$-integral and Riemann-Liouville fractional $\Delta$-derivative on general time scales are also given. In Section 4, we present the properties of fractional $\Delta$-integrals and fractional $\Delta$-differential on time scales. Then in Section 5, Cauchy type problem with Riemann-Liouville fractional $\Delta$-derivative is discussed. In Section 6, for the Riemann-Liouville fractional $\Delta$-differential initial value problem, we discuss the dependency of the solution upon the initial value. In Section 7, by applying the Laplace transform method, we derive explicit solutions to homogeneous fractional $\Delta$-differential equations with constant coefficients. In Section 8, we also use the Laplace transform method to find particular solutions and general 
solutions of the corresponding fractional $\Delta$-differential nonhomogeneous equations.

\section{Preliminaries}

First, we present some preliminaries about time scales in [12].

Definition 1 (see [12]). A time scale $\mathbb{T}$ is a nonempty closed subset of the real numbers. Throughout this paper, $\mathbb{T}$ or $\mathbb{T}_{i}(i=1,2, \ldots, n)$ denotes a time scale.

Definition 2 (see [12]). Let $\mathbb{T}$ be a time scale. For $t \in \mathbb{T}$ one defines the forward jump operator $\sigma: \mathbb{T} \rightarrow \mathbb{T}$ by $\sigma(t):=$ $\inf \{s \in \mathbb{T}: s>t\}$, while the backward jump operator $\rho: \mathbb{T} \rightarrow$ $\mathbb{T}$ is defined by $\rho(t):=\sup \{s \in \mathbb{T}: s<t\}$. If $\sigma(t)>t$, one says that $t$ is right-scattered, while if $\rho(t)<t$, one says that $t$ is leftscattered. Points that are right-scattered and left-scattered at the same time are called isolated. Also, if $t<\sup \mathbb{T}$ and $\sigma(t)=$ $t$, then $t$ is called right-dense, and if $t>\inf \mathbb{T}$ and $\rho(t)=t$, then $t$ is called left-dense.

Definition 3 (see [12]). A function $f: \mathbb{T} \rightarrow \mathbb{R}$ is called regulated provided that its right-sided limits exist (finite) at all right-dense points in $\mathbb{T}$ and its left-sided limits exist (finite) at all left-dense points in $\mathbb{T}$.

Definition 4 (see [12]). Let $h_{k}: \mathbb{T}^{2} \rightarrow \mathbb{R}, k \in \mathbb{N}_{0}$ be defined by

$$
h_{0}(t, s)=1 \quad \forall t, s \in \mathbb{T}
$$

and then recursively by

$$
h_{k+1}(t, s)=\int_{s}^{t} h_{k}(\tau, s) \Delta \tau \quad \forall t, s \in \mathbb{T} .
$$

Definition 5 (see [12]). One defines the Cauchy function $y$ : $\mathbb{T} \times \mathbb{T}^{k^{n}} \rightarrow \mathbb{R}$ for the linear dynamic equation

$$
L y=0, \quad \text { where } L y=y^{\Delta^{n}}+\sum_{i=1}^{n} p_{i} y^{\Delta^{n-i}}
$$

to be for each fixed $s \in \mathbb{T}^{k^{n}}$ the solution of the initial value problem

$$
\begin{gathered}
L y=0, \quad y^{\Delta^{i}}(\sigma(s), s)=0, \quad 0 \leq i \leq n-2, \\
y^{\Delta^{n-1}}(\sigma(s), s)=1 .
\end{gathered}
$$

Remark 6 (see [12]). Note that

$$
y(t, s):=h_{n-1}(t, \sigma(s))
$$

is the Cauchy function of $y^{\Delta^{n}}=0$.

Theorem 7 (variation of constants [12]). Let $f \in C_{\mathrm{rd}}$; then the solution of the initial value problem

$$
L y=f(t), \quad y^{\Delta^{i}}\left(t_{0}\right)=0, \quad 0 \leq i \leq n-1,
$$

is given by

$$
y(t)=\int_{t_{0}}^{t} y(t, s) f(s) \Delta s,
$$

where $y(t, s)$ is the Cauchy function for

$$
L y=0, \quad \text { where } L y=y^{\Delta^{n}}+\sum_{i=1}^{n} p_{i} y^{\Delta^{n-i}} .
$$

Definition 8 (see [2]). The factorial polynomial is defined as

$$
(t)^{(n)}=\prod_{j=0}^{n-1}(t-j)=\frac{\Gamma(t+1)}{\Gamma(t+1-n)} .
$$

For arbitrary $\nu$, define

$$
t^{(v)}=\frac{\Gamma(t+1)}{\Gamma(t+1-v)},
$$

where $\Gamma$ denotes gamma function (see [3]).

Definition 9 (see [12]). One says that a function $p: \mathbb{T} \rightarrow \mathbb{R}$ is regressive provided that

$$
1+\mu(t) p(t) \neq 0
$$

for all $t \in \mathbb{T}^{k}$ holds. The set of all regressive and rd-continuous functions $f: \mathbb{T} \rightarrow \mathbb{R}$ will be denoted by

$$
\mathscr{R}=\mathscr{R}(\mathbb{T})=\mathscr{R}(\mathbb{T}, \mathbb{R}) .
$$

Theorem 10 (see [12]). If $p \in \mathscr{R}$, then the function $\ominus p$ defined by

$$
\ominus p(t):=-\frac{p(t)}{1+\mu(t) p(t)} \quad \forall t \in \mathbb{T}^{k}
$$

is also an element of $\mathscr{R}$.

Definition 11 (see [12]). If $p \in \mathscr{R}$, then one defines the $\Delta$ exponential function by

$$
\begin{aligned}
& e_{p}(t, s) \\
& \quad=\exp \left(\int_{s}^{t} \frac{1}{\mu(\tau)} \log (1+\mu(\tau) p(\tau)) \Delta \tau\right) \quad \text { for } s, t \in \mathbb{T} .
\end{aligned}
$$

Definition 12 (see [12]). If $p \in \mathscr{R}$, then the first order linear dynamic equation

$$
y^{\Delta}=p(t) y
$$

is called regressive.

Theorem 13 (see [12]). Suppose that (15) is regressive and fix $t_{0} \in \mathbb{T}$. Then $e_{p}\left(\cdot, t_{0}\right)$ is a solution of the initial value problem

$$
y^{\Delta}=p(t) y, \quad y\left(t_{0}\right)=1
$$

on $\mathbb{T}$. 
Theorem 14 (see [12]). If $p \in \mathscr{R}$, then

$$
e_{p}(\sigma(t), s):=e_{p}^{\sigma}(t, s)=(1+\mu(t) p(t)) e_{p}(t, s)
$$

Definition 15 (see [12]). Assume that $x: \mathbb{T} \rightarrow \mathbb{R}$ is regulated. Then the $\Delta$-Laplace transform of $x$ is defined by

$$
\mathscr{L}\{x\}\left(z, t_{0}\right)=\int_{t_{0}}^{\infty} x(t) e_{\ominus z}^{\sigma}\left(t, t_{0}\right) \Delta t
$$

for $z \in \mathscr{D}\{x\}$, where $\mathscr{D}\{x\}$ consists of all complex numbers $z \in \mathbb{C}$ for which the improper integral exists.

Definition 16 (uniqueness of the inverse [12]). If the functions $f: \mathbb{T} \rightarrow \mathbb{R}$ and $g: \mathbb{T} \rightarrow \mathbb{R}$ have the same Laplace transform, then $f=g$.

In order to give fractional integral and derivative on a time scale, we need to define fractional power function $h_{\alpha}(t, s)$ which is derived by the inverse of Laplace transform and is introduced in the following section. Before this, we need definitions of shift and convolution and some properties about convolution, such as convolution theorem and associativity, which are introduced in [18].

Definition 17 (see [18]). Let $\mathbb{T}$ be a time scale that sup $\mathbb{T}=\infty$ and fix $t_{0} \in \mathbb{T}$. For a given $f:\left[t_{0}, \infty\right)_{\mathbb{T}} \rightarrow \mathbb{C}$, the solution of the shifting problem

$$
\begin{gathered}
u^{\Delta_{t}}(t, \sigma(s))=-u^{\Delta s}(t, s), \quad t, s \in \mathbb{T}, t \geq s \geq t_{0} \\
u\left(t, t_{0}\right)=f(t), \quad t \in \mathbb{T}, t \geq t_{0}
\end{gathered}
$$

is denoted by $\widehat{f}$ and is called the shift (or delay) of $f$.

Example 18 (see [18]). Consider $\widehat{h_{k}(\cdot, r)}(t, s)=h_{k}(t, s)$ for all $t, s \in \mathbb{T}$, independent of $r$.

Definition 19 (see [18]). For given functions $f, g: \mathbb{T} \rightarrow \mathbb{R}$, their convolution $f * g$ is defined by

$$
(f * g)(t)=\int_{t_{0}}^{t} \widehat{f}(t, \sigma(s)) g(s) \Delta s
$$

where $\widehat{f}$ is the shift of $f$ introduced in Definition 17 .

Theorem 20 (associativity of the convolution [18]). The convolution is associative; that is,

$$
(f * g) * h=f *(g * h) .
$$

Theorem 21 (see [18]). If $f$ is delta differentiable, then

$$
(f * g)^{\Delta}=f^{\Delta} * g+f\left(t_{0}\right) g,
$$

and if $g$ is delta differentiable, then

$$
(f * g)^{\Delta}=f * g^{\Delta}+f g\left(t_{0}\right) .
$$

Theorem 22 (see [18]). If $f$ and $g$ are infinitely often $\Delta$ differentiable, then for all $k \in N_{0}$

$$
\begin{aligned}
(f * g)^{\Delta^{k}} & =f^{\Delta^{k}} * g+\sum_{\nu=0}^{k-1} f^{\Delta^{\nu}}\left(t_{0}\right) g^{\Delta^{k-1-\gamma}} \\
& =f * g^{\Delta^{k}}+\sum_{\nu=0}^{k-1} f^{\Delta^{\nu}} g^{\Delta^{k-1-\nu}}\left(t_{0}\right), \\
(f * g)^{\Delta^{k}}\left(t_{0}\right) & =\sum_{\nu=0}^{k-1} f^{\Delta^{\nu}}\left(t_{0}\right) g^{\Delta^{k-1-\gamma}}\left(t_{0}\right) .
\end{aligned}
$$

Theorem 23 (convolution theorem [18]). Suppose that $f, g$ : $\mathbb{T} \rightarrow \mathbb{R}$ are locally $\Delta$-integrable functions on $\mathbb{T}$ and their convolution $f * g$ is defined by (20). Then,

$$
\begin{aligned}
\mathscr{L} & \{f * g\}(z) \\
& =\mathscr{L}\{f\}(z) \cdot \mathscr{L}\{g\}(z), \quad z \in \mathscr{D}\{f\} \cap \mathscr{D}\{g\} .
\end{aligned}
$$

Theorem 24 (see [12]). Assume that $x: \mathbb{T} \rightarrow \mathbb{C}$ is a mapping, such that $x^{\Delta^{k}}$ is regulated. Then

$$
\mathscr{L}\left\{x^{\Delta^{k}}\right\}\left(z, t_{0}\right)=z^{k} \mathscr{L}\{x\}\left(z, t_{0}\right)-\sum_{i=0}^{k-1} z^{k-i-1} x^{\Delta^{i}}\left(t_{0}\right)
$$

for those regressive $z \in \mathbb{C}$ satisfying

$$
\lim _{t \rightarrow \infty}\left\{x^{\Delta^{i}}(t) e_{\ominus z}\left(t, t_{0}\right)\right\}=0, \quad i=0,1, \ldots, k-1 .
$$

Theorem 25 (see [12]). Assume that $h_{k}\left(t, t_{0}\right), k \in \mathbb{N}_{0}$ are defined as in Definition 4. Then

$$
\mathscr{L}\left\{h_{k}\left(\cdot, t_{0}\right)\right\}\left(z, t_{0}\right)=\frac{1}{z^{k+1}}
$$

for those regressive $z \in \mathbb{C}$ satisfying

$$
\lim _{t \rightarrow \infty}\left\{h_{k}\left(t, t_{0}\right) e_{\ominus z}\left(t, t_{0}\right)\right\}=0 .
$$

\section{3. $\Delta$-Power Function and Fractional Integral and Derivative on Time Scales}

In this section, inspired by property of $h_{k}\left(\cdot, t_{0}\right)$ in Theorem 25 for $k \in \mathbb{N}_{0}$, we define fractional $\Delta$-power functions $h_{\alpha}(t, s)$ for $\alpha \in \mathbb{R}$ by using inversion of $\Delta$-Laplace transform and give definitions of fractional integral and derivative on time scales.

Definition 26. One defines fractional generalized $\Delta$-power function $h_{\alpha}\left(t, t_{0}\right)$ on time scales

$$
h_{\alpha}\left(t, t_{0}\right)=\mathscr{L}^{-1}\left\{\frac{1}{z^{\alpha+1}}\right\}(t)
$$

to those suitable regressive $z \in \mathbb{C} \backslash\{0\}$ such that $\mathscr{L}^{-1}$ exist for $\alpha \in \mathbb{R}, t \geq t_{0}$. Fractional generalized $\Delta$-power function $h_{\alpha}(t, s)$ on time scales is defined as the shift of $h_{\alpha}\left(t, t_{0}\right)$; that is,

$$
\left.h_{\alpha}(t, s)=\widehat{h_{\alpha}\left(\cdot, t_{0}\right.}\right)(t, s) \quad\left(t \geq s \geq t_{0}\right) .
$$


Applying the initial value theorem of Laplace transform (see, e.g., [15, Theorem 1.3], for $\alpha>0$, we have

$$
h_{\alpha}\left(t_{0}, t_{0}\right)=\lim _{z \rightarrow \infty} z \cdot \frac{1}{z^{\alpha+1}}=0 .
$$

Theorem 27. For $\alpha, \beta \in \mathbb{R}$, one has

$$
\left(h_{\alpha}\left(\cdot, t_{0}\right) * h_{\beta}\left(\cdot, t_{0}\right)\right)(t)=h_{\alpha+\beta+1}\left(t, t_{0}\right) .
$$

Proof. According to convolution theorem,

$$
\begin{aligned}
\mathscr{L} & \left\{\left(h_{\alpha}\left(\cdot, t_{0}\right) * h_{\beta}\left(\cdot, t_{0}\right)\right)(t)\right\}\left(z, t_{0}\right) \\
& =\mathscr{L}\left\{h_{\alpha}\left(t, t_{0}\right)\right\}\left(z, t_{0}\right) \mathscr{L}\left\{h_{\beta}\left(t, t_{0}\right)\right\}\left(z, t_{0}\right) \\
& =\frac{1}{z^{\alpha+1}} \cdot \frac{1}{z^{\beta+1}}=\frac{1}{z^{\alpha+\beta+2}} \\
& =\mathscr{L}\left\{h_{\alpha+\beta+1}\left(t, t_{0}\right)\right\}\left(z, t_{0}\right) .
\end{aligned}
$$

By the uniqueness of inverse transform for Laplace transform, we obtain

$$
\begin{aligned}
& \left(h_{\alpha}\left(\cdot, t_{0}\right) * h_{\beta}\left(\cdot, t_{0}\right)\right)(t) \\
& \left.=\int_{t_{0}}^{t} \widehat{h_{\alpha}\left(\cdot, t_{0}\right.}\right)(t, \sigma(\tau)) h_{\beta}(\tau, s) \Delta \tau=h_{\alpha+\beta+1}\left(t, t_{0}\right) .
\end{aligned}
$$

Moreover, if we take $\alpha=0$, then

$$
\left(1 * h_{\beta}\right)\left(t, t_{0}\right)=\int_{t_{0}}^{t} h_{\beta}(\tau, s) \Delta \tau=h_{\beta+1}\left(t, t_{0}\right) .
$$

That is,

$$
h_{\beta+1}^{\Delta}\left(t, t_{0}\right)=h_{\beta}\left(t, t_{0}\right) .
$$

Now, we will give the definitions of fractional $\Delta$-integral and $\Delta$-derivative which are the main context in this section.

Definition 28. Let $\Omega$ be a finite interval on a time scale $\mathbb{T}$, $t_{0}, t \in \Omega$. For $\alpha \geq 0$ and for a function $f: \mathbb{T} \rightarrow \mathbb{R}$, the Riemann-Liouville fractional $\Delta$-integral of order $\alpha$ is defined by $I_{\Delta, t_{0}}^{0} f(t)=f(t)$ and

$$
\begin{aligned}
\left(I_{\Delta, t_{0}}^{\alpha} f\right)(t) & =\left(h_{\alpha-1}\left(\cdot, t_{0}\right) * f\right)(t) \\
& \left.=\int_{t_{0}}^{t} \widehat{h_{\alpha-1}\left(\cdot, t_{0}\right.}\right)(t, \sigma(\tau)) f(\tau) \Delta \tau \\
& =\int_{t_{0}}^{t} h_{\alpha-1}(t, \sigma(\tau)) f(\tau) \Delta \tau,
\end{aligned}
$$

for $\alpha>0, t>t_{0}$.

When $\mathbb{T}=\mathbb{R}, h_{\alpha-1}(x, a)=(x-a)^{\alpha-1} / \Gamma(\alpha)$, according to Definition 17,

$$
\widehat{h_{\alpha-1}(\cdot, a)}(x, t)=\frac{(x-t)^{\alpha-1}}{\Gamma(\alpha)}
$$

satisfy

$$
\begin{gathered}
\frac{\partial h_{\alpha-1}(\cdot, a)}{\partial x}(x, t) \\
\widehat{h_{\alpha-1}(\cdot, a)}(x, a)=-\frac{\partial \widehat{h_{\alpha-1}(\cdot, a)}(x, t)}{\partial t} \\
h_{\alpha-1}(x, a) .
\end{gathered}
$$

When $\mathbb{T}=\mathbb{Z}, h_{\alpha-1}(x, a)=(x-a)^{(\alpha-1)} / \Gamma(\alpha)$, according to Definition 17,

$$
\widehat{h_{\alpha-1}(\cdot, a)}(x, t)=\frac{(x-t)^{(\alpha-1)}}{\Gamma(\alpha)}
$$

satisfy

$$
\begin{gathered}
h_{\alpha-1}(\cdot, a)(x+1, t+1)-\widehat{h_{\alpha-1}(\cdot, a)}(x, t+1) \\
=-\widehat{h_{\alpha-1}(\cdot, a)}(x, t+1)+\widehat{h_{\alpha-1}(\cdot, a)}(x, t), \\
\widehat{h_{\alpha-1}(\cdot, a)}(x, a)=h_{\alpha-1}(x, a) .
\end{gathered}
$$

As an especial case of Definition 28, we have the following examples.

Example 29 (see [3]). When $\mathbb{T}=\mathbb{R}$, the fractional $\Delta$-integral of order $\alpha$ is defined by

$$
\left(I_{a+}^{\alpha} f\right)(x):=\frac{1}{\Gamma(\alpha)} \int_{a}^{x} \frac{1}{(x-t)^{1-\alpha}} f(t) d t \quad(x>a) .
$$

Example 30. When $\mathbb{T}=\mathbb{Z}$, Consider the following.

(1) The $n$th integral of $f$ is defined by

$$
{ }_{a} \Delta^{-n} x(t)=\frac{1}{\Gamma(n)} \sum_{s=a}^{t-n}(t-\sigma(s))^{(n-1)} x(s) .
$$

Here $t-n \geq a, \sigma(s)=s+1$.

Note that power function $(t-\sigma(s))^{(n-1)} / \Gamma(n)$ vanishes at $s \geq t-n+1$. So

$$
\begin{aligned}
{ }_{a} \Delta^{-n} x(t) & =\frac{1}{\Gamma(n)} \sum_{s=a}^{t-1}(t-\sigma(s))^{(n-1)} x(s) \\
& =\frac{1}{\Gamma(n)} \sum_{s=a}^{t-n}(t-\sigma(s))^{(n-1)} x(s), \quad \text { for } t-n \geq a
\end{aligned}
$$

(2) The $\gamma$ th fractional sum of $f$ is defined by

$$
\begin{array}{r}
{ }_{a} \Delta^{-\gamma} x(t)=\frac{1}{\Gamma(\gamma)} \sum_{s=a}^{t-1}(t-\sigma(s))^{(\gamma-1)} x(s), \\
\text { for } t \geq a-[-\gamma] .
\end{array}
$$

Definition 31. Let $\alpha \geq 0, m=[\alpha]+1$, and $f: \mathbb{T} \rightarrow \mathbb{R}$. For $s, t \in \mathbb{T}^{k^{m}}$ with $s<t$, the Riemann-Liouville fractional $\Delta$-derivative of order $\alpha$ is defined by the expression

$$
D_{\Delta, s}^{\alpha} f(t):=D_{\Delta}^{m} I_{\Delta, s}^{m-\alpha} f(t),
$$

if it exists. 
Throughout this paper, we denote $f^{\Delta^{n}}=D_{\Delta}^{n} f=D_{\Delta, s}^{n} f$, $n \in \mathbb{N}$, and, for $\alpha<0, D_{\Delta, s}^{\alpha} f(t)$ means $I_{\Delta, s}^{-\alpha} f(t)$ and $I_{\Delta, s}^{\alpha} f(t)$ means $D_{\Delta, s}^{-\alpha} f(t)$.

Finally, we present the definition of $\Delta$-Mittag-Leffler function which is an important tool for solving fractional difference equation.

Definition 32. $\Delta$-Mittag-Leffler function is defined by

$$
{ }_{\Delta} F_{\alpha, \beta}\left(\lambda, t, t_{0}\right)=\sum_{j=0}^{\infty} \lambda^{j} h_{j \alpha+\beta-1}\left(t, t_{0}\right),
$$

provided that the right series is convergent, where $\alpha, \beta>0$, $\lambda \in \mathbb{R}$.

Example 33. When $0<\lambda<1$, for any $j,\left|h_{j \alpha+\beta-1}\left(t, t_{0}\right)\right|<$ $M$, we can obtain that the series $\sum_{j=0}^{\infty} \lambda^{j} h_{j \alpha+\beta-1}\left(t, t_{0}\right)$ is convergent.

Example 34. When $\mathbb{T}=\mathbb{R}, \sum_{j=0}^{\infty} \lambda^{j} h_{j \alpha+\beta-1}\left(t, t_{0}\right)=$ $\sum_{j=0}^{\infty} \lambda^{j}\left(\left(t-t_{0}\right)^{j \alpha+\beta-1} / \Gamma(j \alpha+\beta)\right)$. Since $\sum_{j=0}^{\infty} \lambda^{j}\left(\left(t-t_{0}\right)^{j \alpha+\beta-1} /\right.$ $\Gamma(j \alpha+\beta))$ is convergent for any $t \geq t_{0}, \sum_{j=0}^{\infty} \lambda^{j} h_{j \alpha+\beta-1}\left(t, t_{0}\right)$ is convergent. That is, ${ }_{\Delta} F_{\alpha, \beta}\left(\lambda, t, t_{0}\right)$ is defined as $t \geq t_{0}$.

As to the Laplace transform of $\Delta$-Mittag-Leffler function, we have the following theorem.

Theorem 35. The Laplace transform of $\Delta$-Mittag-Leffler function is

$$
\mathscr{L}\left\{{ }_{\Delta} F_{\alpha, \beta}\left(\lambda, t, t_{0}\right)\right\}\left(z, t_{0}\right)=\frac{z^{\alpha-\beta}}{z^{\alpha}-\lambda} \quad\left(|\lambda|<|z|^{\alpha}\right) .
$$

Proof. According to the definition of Laplace transform, it is obtained that

$$
\begin{aligned}
\mathscr{L} & \left\{{ }_{\Delta} F_{\alpha, \beta}\left(\lambda, t, t_{0}\right)\right\}\left(z, t_{0}\right) \\
& =\int_{t_{0} \Delta}^{\infty} F_{\alpha, \beta}\left(\lambda, t, t_{0}\right) \cdot e_{\ominus z}^{\sigma}\left(t, t_{0}\right) \Delta t \\
& =\int_{t_{0}}^{\infty} \sum_{j=0}^{\infty} \lambda^{j} h_{j \alpha+\beta-1}\left(t, t_{0}\right) \cdot e_{\ominus z}^{\sigma}\left(t, t_{0}\right) \Delta t \\
& =\sum_{j=0}^{\infty} \lambda^{j} \int_{t_{0}}^{\infty} h_{j \alpha+\beta-1}\left(t, t_{0}\right) \cdot e_{\ominus z}^{\sigma}\left(t, t_{0}\right) \Delta t \\
& =\sum_{j=0}^{\infty} \lambda^{j} \mathscr{L}\left\{h_{j \alpha+\beta-1}\left(t, t_{0}\right)\right\}\left(z, t_{0}\right) \\
& =\sum_{j=0}^{\infty} \lambda^{j} \frac{1}{z^{j \alpha+\beta}}=z^{-\beta} \sum_{j=0}^{\infty} \lambda^{j}\left(z^{-\alpha}\right)^{j} \\
& =\frac{z^{\alpha-\beta}}{z^{\alpha}-\lambda} \quad\left(|\lambda|<|z|^{\alpha}\right) .
\end{aligned}
$$

By differentiating $k$ times with respect to $\lambda$ on both sides of the formula in Theorem 35, we get the following result:

$$
\mathscr{L}\left\{\frac{\partial^{k}}{\partial \lambda^{k}}{ }_{\Delta} F_{\alpha, \beta}\left(\lambda, t, t_{0}\right)\right\}\left(z, t_{0}\right)=\frac{k ! z^{\alpha-\beta}}{\left(z^{\alpha}-\lambda\right)^{k+1}} .
$$

\section{Properties of Fractional $\Delta$-Integral and $\Delta$-Derivative on Time Scales}

In this section, we mainly give the properties of fractional $\Delta$ integral and $\Delta$-derivative on time scales which are needed in the following sections.

Theorem 36. Let $\alpha>0, m=[a]+1$, and $\beta \in \mathbb{R}$. Then

$$
\begin{aligned}
\text { (1) } I_{\Delta, t_{0}}^{\alpha} h_{\beta-1}\left(t, t_{0}\right) & =h_{\beta+\alpha-1}\left(t, t_{0}\right), \\
\text { (2) } D_{\Delta, t_{0}}^{\alpha} h_{\beta-1}\left(t, t_{0}\right) & =h_{\beta-\alpha-1}\left(t, t_{0}\right) .
\end{aligned}
$$

Proof. (1) According to Definition 28 and Theorem 27, we have

$$
\begin{aligned}
& I_{\Delta, t_{0}}^{\alpha} h_{\beta-1}\left(t, t_{0}\right) \\
& \quad=\left(h_{\alpha-1}\left(\cdot, t_{0}\right) * h_{\beta-1}\left(\cdot, t_{0}\right)\right)(t)=h_{\alpha+\beta-1}\left(t, t_{0}\right) .
\end{aligned}
$$

(2) By Definition 31, it is obtained that

$$
D_{\Delta, t_{0}}^{\alpha} h_{\beta-1}\left(t, t_{0}\right)=D_{\Delta}^{m} I_{\Delta, t_{0}}^{m-\alpha} h_{\beta-1}\left(t, t_{0}\right)=D^{m} h_{m+\beta-\alpha-1}\left(t, t_{0}\right) \text {. }
$$

Then

$$
D^{m} h_{m+\beta-\alpha-1}\left(t, t_{0}\right)=h_{\beta-\alpha-1}\left(t, t_{0}\right) .
$$

In particular, if $\beta=1, \alpha>0$, then the Riemann-Liouville fractional $\Delta$-derivatives of a constant are, in general, not equal to zero:

$$
D_{\Delta, t_{0}}^{\alpha} 1=h_{-\alpha}\left(t, t_{0}\right) \quad(0<\alpha<1) .
$$

On the other hand, for $j=1,2, \ldots, m$,

$$
D_{\Delta, t_{0}}^{\alpha} h_{\alpha-j}(t, s)=0 .
$$

In fact,

$$
\begin{aligned}
D_{\Delta, t_{0}}^{\alpha} h_{\alpha-j}(t, s) & =D_{\Delta}^{m} I_{\Delta, t_{0}}^{m-\alpha} h_{\alpha-j}(t, s) \\
& =D_{\Delta}^{m} h_{m-j}(t, s)=0 .
\end{aligned}
$$

From Theorem 36, we derive the following result in [3] when $\mathbb{T}=\mathbb{R}$.

Corollary 37 (see [3]). If $\alpha \geq 0$ and $\beta>0$, then

$$
\begin{aligned}
& \left(I_{a+}^{\alpha}(t-a)^{\beta-1}\right)(x)=\frac{\Gamma(\beta)}{\Gamma(\beta+\alpha)}(x-a)^{\beta+\alpha-1} \quad(\alpha>0), \\
& \left(D_{a+}^{\alpha}(t-a)^{\beta-1}\right)(x)=\frac{\Gamma(\beta)}{\Gamma(\beta-\alpha)}(x-a)^{\beta-\alpha-1} \quad(\alpha \geq 0) .
\end{aligned}
$$


In particular, if $\beta=1$ and $\alpha \geq 0$, then the Riemann-Liouville fractional derivatives of a constant are, in general, not equal to zero:

$$
\left(D_{a+}^{\alpha} 1\right)(x)=\frac{(x-a)^{-\alpha}}{\Gamma(1-\alpha)} \quad(0<\alpha<1) .
$$

On the other hand, for $j=1,2, \ldots,[\alpha]+1$,

$$
\left(D_{a+}^{\alpha}(t-a)^{\alpha-j}\right)(x)=0 .
$$

As to the fractional sum and difference, we have the following result, which is an improvement of Lemma 3.1 in [1].

Corollary 38. Let $\mu \in \mathbb{R} \backslash\{\ldots,-2,-1\}$. Then

(1) $\Delta_{a}^{-v}(t-a)^{(\mu)}=\mu^{(-v)}(t-a)^{(u+v)}$, for $t \geq a-[-\nu]$,

(2) $\Delta_{a}^{v}(t-a)^{(\mu)}=\mu^{(\nu)}(t-a)^{(u-v)}$, for $t \geq a+1$.

Lemma 39 (Taylor's formula). Let $n \in \mathbb{N}$. Suppose that the function $f$ is $n$ times differentiable on $\mathbb{T}^{k^{n}}$. Let $\alpha \in \mathbb{T}^{k^{n-1}}, t \in \mathbb{T}$, and $t>\alpha$. Then one has

$$
f(t)=\sum_{k=0}^{n} h_{k}(t, \alpha) f^{\Delta^{k}}(\alpha)+\int_{\alpha}^{t} h_{n}(t, \sigma(\tau)) f^{\Delta^{n+1}}(\tau) \Delta \tau .
$$

Proof. Let $g(t):=f^{\Delta^{n+1}}(t)$. Then $f$ solves the initial value problem

$$
x^{\Delta^{n+1}}=g(t), \quad x^{\Delta^{k}}(\alpha)=f^{\Delta^{k}}(\alpha), \quad 0 \leq k \leq n .
$$

Note that the Cauchy function for $y^{\Delta^{n+1}}=0$ is $y(t, s)=h_{n}(t, \sigma(s))$. By the variation of constants formula in Theorem 7,

$$
f(t)=u(t)+\int_{\alpha}^{t} y(t, \sigma(\tau)) g(\tau) \Delta \tau
$$

where $u$ solves the initial value problem

$$
u^{\Delta^{n+1}}=0, \quad u^{\Delta^{m}}(\alpha)=f^{\Delta^{m}}(\alpha), \quad 0 \leq m \leq n .
$$

To validate the claim that $u(t)=\sum_{k=0}^{n} h_{k}(t, \alpha) f^{\Delta^{k}}(\alpha)$, set

$$
\omega(t):=\sum_{k=0}^{n} h_{k}(t, \alpha) f^{\Delta^{k}}(\alpha) \text {. }
$$

By the properties of $h_{k}(t, \alpha), \omega^{\Delta^{n+1}}(t)=0$. We have moreover that

$$
\omega^{\Delta^{m}}(t)=\sum_{k=m}^{n} h_{k-m}(t, \alpha) f^{\Delta^{k}}(\alpha)
$$

so that

$$
\omega^{\Delta^{m}}(\alpha)=\sum_{k=m}^{n} h_{k-m}(\alpha, \alpha) f^{\Delta^{k}}(\alpha)=f^{\Delta^{m}}(\alpha),
$$

for $0 \leq m \leq n$. We consequently have that $\omega$ also solves (66), whence $u \equiv \omega$ by uniqueness.
Lemma 40. (1) For $\alpha>0, n=[\alpha]+1$, let $f$ be a function which is $n$ times $\Delta$-differentiable on $\mathbb{T}^{k^{n}}$ with $f^{\Delta^{n}}$ rd-continuous over $\mathbb{T}$, and it is valid that

$$
\begin{aligned}
I_{\Delta, t_{0}}^{\alpha} f(t) \\
\quad=\sum_{k=0}^{n-1} h_{k+\alpha}\left(t, t_{0}\right) f^{\Delta^{k}}\left(t_{0}\right)+\left(h_{n+\alpha-1}\left(\cdot, t_{0}\right) * f^{\Delta^{n}}\right)(t) .
\end{aligned}
$$

(2) For $\alpha \geq 0, n=[\alpha]+1$, let $f$ be a function which is $n$ times $\Delta$-differentiable on $\mathbb{T}^{k^{n}}$ with $f^{\Delta^{n}}$ rd-continuous over $\mathbb{T}$ and $D_{\Delta, t_{0}}^{\alpha} f$ exists almost on $\mathbb{T}$, and it is valid that

$$
\begin{aligned}
& D_{\Delta, t_{0}}^{\alpha} f(t) \\
& \quad=\sum_{k=0}^{n-1} h_{k-\alpha}\left(t, t_{0}\right) f^{\Delta^{k}}\left(t_{0}\right)+\left(h_{n-\alpha-1}\left(\cdot, t_{0}\right) * f^{\Delta^{n}}\right)(t) .
\end{aligned}
$$

Proof. By Taylor's formula

$$
f(t)=\sum_{k=0}^{n-1} h_{k}\left(t, t_{0}\right) f^{\Delta^{k}}\left(t_{0}\right)+\int_{t_{0}}^{t} h_{n-1}(t, \sigma(\tau)) f^{\Delta^{n}}(\tau) \Delta \tau
$$

we have

$$
\begin{aligned}
I_{\Delta, t_{0}}^{\alpha} f(t)= & \sum_{k=0}^{n-1} h_{k+\alpha}\left(t, t_{0}\right) f^{\Delta^{k}}\left(t_{0}\right) \\
& +\left(h_{\alpha-1}\left(\cdot, t_{0}\right) *\left(h_{n-1}\left(\cdot, t_{0}\right) * f^{\Delta^{n}}\right)\right)(t) \\
= & \sum_{k=0}^{n-1} h_{k+\alpha}\left(t, t_{0}\right) f^{\Delta^{k}}\left(t_{0}\right) \\
& +\left(\left(h_{\alpha-1}\left(\cdot, t_{0}\right) * h_{n-1}\left(\cdot, t_{0}\right)\right) * f^{\Delta^{n}}\right)(t) \\
= & \sum_{k=0}^{n-1} h_{k+\alpha}\left(t, t_{0}\right) f^{\Delta^{k}}\left(t_{0}\right) \\
& +\left(h_{\alpha+n-1}\left(\cdot, t_{0}\right) * f^{\Delta^{n}}\right)(t) .
\end{aligned}
$$

Besides,

$$
\begin{aligned}
D_{\Delta, t_{0}}^{\alpha} f(t)= & D_{\Delta}^{m} I_{\Delta, t_{0}}^{m-\alpha} f(t) \\
= & D_{\Delta}^{m}\left[\sum_{k=0}^{n-1} h_{k+m-\alpha}\left(t, t_{0}\right) f^{\Delta^{k}}\left(t_{0}\right)\right. \\
& \left.+\left(h_{m+n-\alpha-1}\left(\cdot, t_{0}\right) * f^{\Delta^{n}}\right)(t)\right] \\
& =\sum_{k=0}^{n-1} h_{k-\alpha}\left(t, t_{0}\right) f^{\Delta^{k}}\left(t_{0}\right)+\left(h_{n-\alpha-1}\left(\cdot, t_{0}\right) * f^{\Delta^{n}}\right)(t),
\end{aligned}
$$


where

$$
\begin{aligned}
D_{\Delta}^{m} & \left(h_{m+n-\alpha-1}\left(\cdot, t_{0}\right) * f^{\Delta^{n}}\right)(t) \\
& =\left(h_{n-\alpha-1}\left(\cdot, t_{0}\right) * f^{\Delta^{n}}\right)(t)+\sum_{k=0}^{m} h_{m+n-\alpha-1-k}\left(t_{0}, t_{0}\right) g^{\Delta^{m-k}} \\
& =\left(h_{n-\alpha-1}\left(\cdot, t_{0}\right) * f^{\Delta^{n}}\right)(t) .
\end{aligned}
$$

When $\mathbb{T}=\mathbb{R}$, there is the following corollary.

Corollary 41 (see [3]). Let $\alpha \geq 0$ and $n=[\alpha]+1$. If $y(x) \in A C^{n}[a, b]$, then the fractional derivative $D_{a+}^{\alpha} y$ exists almost everywhere on $[a, b]$ and can be represented in the form

$$
\begin{aligned}
\left(D_{a+}^{\alpha} y\right)(x)= & \sum_{k=0}^{n-1} \frac{y^{(k)}(a)}{\Gamma(1+k-\alpha)}(x-a)^{k-\alpha} \\
& +\frac{1}{\Gamma(n-\alpha)} \int_{a}^{x} \frac{y^{(n)}(t) d t}{(x-t)^{\alpha-n+1}} .
\end{aligned}
$$

Theorem 42. For $\alpha>0$ and $\beta>0$, then $\left(I_{\Delta, t_{0}}^{\alpha} I_{\Delta, t_{0}}^{\beta} f\right)(t)=$ $\left(I_{\Delta, t_{0}}^{\alpha+\beta} f\right)(t)$.

Proof. According to Definition 28, Theorem 20 and 27,

$$
\begin{aligned}
\left(I_{\Delta, t_{0}}^{\alpha} I_{\Delta, t_{0}}^{\beta} f\right)(t) & =\left(h_{\alpha-1}\left(\cdot, t_{0}\right) *\left(h_{\beta-1}\left(\cdot, t_{0}\right) * f\right)\right)(t) \\
& =\left(\left(h_{\alpha-1}\left(\cdot, t_{0}\right) * h_{\beta-1}\left(\cdot, t_{0}\right)\right) * f\right)(t) \\
& =\left(h_{\alpha+\beta-1}\left(\cdot, t_{0}\right) * f\right)(t)=\left(I_{\Delta, t_{0}}^{\alpha+\beta} f\right)(t) .
\end{aligned}
$$

Theorem 43. For $\alpha>0, n$ is a positive integer; if $f$ is $\Delta$ differentiable and the highest order derivative is rd-continuous over $\mathbb{T}$, then it is valid that

$$
\begin{aligned}
& \text { (1) } D_{\Delta}^{n} D_{\Delta, t_{0}}^{\alpha} f(t)=D_{\Delta, t_{0}}^{n+\alpha} f(t), \\
& \text { (2) } D_{\Delta}^{n} I_{\Delta, t_{0}}^{\alpha} f(t)=I_{\Delta, t_{0}}^{\alpha-n} f(t) .
\end{aligned}
$$

Proof. (1) Suppose that $f$ is a function which is $s$ times $\Delta$-differentiable on $\mathbb{T}^{k^{s}}$ with $f^{\Delta^{s}}$ rd-continuous over $\mathbb{T}$. By Lemma 40(2),

$$
\begin{aligned}
D_{\Delta}^{n} & D_{\Delta, t_{0}}^{\alpha} f(t) \\
& =D_{\Delta}^{n}\left[\sum_{k=0}^{s-1} h_{k-\alpha}\left(t, t_{0}\right) f^{\Delta^{k}}\left(t_{0}\right)+\left(h_{s-1-\alpha}\left(\cdot, t_{0}\right) * f^{\Delta^{s}}\right)(t)\right] \\
& =\sum_{k=0}^{s-1} h_{k-\alpha-n}\left(t, t_{0}\right) f^{\Delta^{k}}\left(t_{0}\right)+\left(h_{s-1-\alpha-n}\left(\cdot, t_{0}\right) * f^{\Delta^{s}}\right)(t) \\
& =D_{\Delta, t_{0}}^{n+\alpha} f(t) .
\end{aligned}
$$

By a similar way, we can get (2).

When $\mathbb{T}=\mathbb{Z}$, we have the following corollary.
Corollary 44. Let $f: \mathbb{N}_{a} \rightarrow \mathbb{R}$ be given. For any $k \in \mathbb{N}_{0}$ and $\mu>0$ with $M-1<\mu \leq M$, one has

$$
\begin{gathered}
\Delta^{k} \Delta_{a}^{-\mu} f(t)=\Delta_{a}^{k-\mu} f(t), \quad \text { for } t \geq a-[-\mu], \\
\Delta^{k} \Delta_{a}^{\mu} f(t)=\Delta_{a}^{k+\mu} f(t), \quad \text { for } t \geq a+1 .
\end{gathered}
$$

Theorem 45. For $\alpha>0, n$ is a positive integer; if $f$ is $\Delta$ differentiable and the highest order derivative is rd-continuous over $\mathbb{T}$, then it is valid that

(1) $D_{\Delta, t_{0}}^{n+\alpha} f(t)=D_{\Delta, t_{0}}^{\alpha} D_{\Delta, t_{0}}^{n} f(t)+\sum_{k=0}^{n-1} h_{k-\alpha-n}\left(t, t_{0}\right) f^{\Delta^{k}}\left(t_{0}\right)$,

(2) $D_{\Delta, t_{0}}^{n-\alpha} f(t)=I_{\Delta, t_{0}}^{\alpha} D_{\Delta, t_{0}}^{n} f(t)+\sum_{k=0}^{n-1} h_{k+\alpha-n}\left(t, t_{0}\right) f^{\Delta^{k}}\left(t_{0}\right)$.

Proof. (1) In the proof of Theorem 43(1), if we take $s=n+m$, then we have

$$
\begin{aligned}
D_{\Delta}^{n} D_{\Delta, t_{0}}^{\alpha} f(t)= & D_{\Delta, t_{0}}^{n+\alpha} f(t) \\
= & \sum_{k=0}^{n+m-1} h_{k-\alpha-n}\left(t, t_{0}\right) f^{\Delta^{k}}\left(t_{0}\right) \\
& +\left(h_{s-1-\alpha-n}\left(\cdot, t_{0}\right) * f^{\Delta^{s}}\right)(t) .
\end{aligned}
$$

As $D_{\Delta}^{n} f(t)$ is $m$ times $\Delta$-differentiable on $\mathbb{T}^{k^{m+n}}$, we have

$$
\begin{aligned}
D_{\Delta, t_{0}}^{\alpha}\left(D_{\Delta}^{n} f(t)\right)= & \sum_{k=0}^{m-1} h_{k-\alpha}\left(t, t_{0}\right) f^{\Delta^{n+k}}\left(t_{0}\right) \\
& +\left(h_{-\alpha+m-1}\left(\cdot, t_{0}\right) * f^{\Delta^{s}}\right)(t) .
\end{aligned}
$$

By (82) and (83), if $f$ is at least $n$ times $\Delta$-differentiable with the highest order derivative rd-continuous over $\mathbb{T}$, then we have

$$
\begin{aligned}
D_{\Delta}^{n} D_{\Delta, t_{0}}^{\alpha} f(t)= & D_{\Delta, t_{0}}^{\alpha}\left(D_{\Delta}^{n} f(t)\right) \\
& +\sum_{k=0}^{n-1} h_{-\alpha-n+k}\left(t, t_{0}\right) f^{\Delta^{k}}\left(t_{0}\right) .
\end{aligned}
$$

Thus

$$
D_{\Delta}^{n} D_{\Delta, t_{0}}^{\alpha} f(t)=D_{\Delta, t_{0}}^{\alpha}\left(D_{\Delta}^{n} f(t)\right)
$$

is valid if and only if

$$
f^{\Delta^{k}}\left(t_{0}\right)=0, \quad k=0, \ldots, n-1
$$


(2) Similarly, we have

$$
\begin{aligned}
I_{\Delta, t_{0}}^{\alpha} D_{\Delta}^{n} f(t)= & \sum_{k=0}^{m-1} h_{k+\alpha}\left(t, t_{0}\right) f^{\Delta^{k+n}}\left(t_{0}\right) \\
& +\left(h_{\alpha+m-1}\left(\cdot, t_{0}\right) * f^{\Delta^{m+n}}\right)(t), \\
D_{\Delta}^{n} I_{\Delta, t_{0}}^{\alpha} f(t)= & I_{\Delta, t_{0}}^{\alpha-n} f(t) \\
= & \sum_{k=0}^{n+m-1} h_{k+\alpha-n}\left(t, t_{0}\right) f^{\Delta^{k}}\left(t_{0}\right) \\
& +\left(h_{\alpha+m-1}\left(\cdot, t_{0}\right) * f^{\Delta^{m+n}}\right)(t) .
\end{aligned}
$$

Therefore

$$
D_{\Delta}^{n} I_{\Delta, t_{0}}^{\alpha} f(t)=I_{\Delta, t_{0}}^{\alpha} D_{\Delta}^{n} f(t)+\sum_{k=0}^{n-1} h_{k+\alpha-n}\left(t, t_{0}\right) f^{\Delta^{k}}\left(t_{0}\right)
$$

Provided that $f$ is at least $n$ times $\Delta$-differentiable with the highest order derivative rd-continuous over $\mathbb{T}$.

Thus

$$
D_{\Delta}^{n} I_{\Delta, t_{0}}^{\alpha} f(t)=I_{\Delta, t_{0}}^{\alpha} D_{\Delta}^{n} f(t)
$$

is valid if and only if

$$
f^{\Delta^{k}}\left(t_{0}\right)=0, \quad k=0, \ldots, n-1
$$
$\mathbb{Z}$.

In particular, there are corollaries for $\mathbb{T}=\mathbb{R}$ and for $\mathbb{T}=$

Corollary 46 (see [3]). Let $\alpha>0$ and $\beta>0$ be such that $n-1<\alpha \leq n, m-1<\beta \leq m(n, m \in \mathbb{N})$ and $\alpha+\beta<n$, and let $f \in L_{1}(a, b)$ and $f_{m-\alpha} \in A C^{m}([a, b])$. Then one has the following index rule:

$$
\begin{aligned}
\left(D_{a+}^{\alpha} D_{a+}^{\beta} f\right)(x)= & \left(D_{a+}^{\alpha+\beta} f\right)(x) \\
& -\sum_{j=1}^{m}\left(D_{a+}^{\beta-j} f\right)(a+) \frac{(x-a)^{-j-\alpha}}{\Gamma(1-j-\alpha)} .
\end{aligned}
$$

Corollary 47. Let $f: \mathbb{N}_{a} \rightarrow \mathbb{R}$ be given. For any $k \in \mathbb{N}_{0}$ and $v>0$,

$$
\begin{array}{r}
\Delta_{a}^{-v} \Delta^{k} f(t)=\Delta_{a}^{k-v} f(t)-\sum_{j=0}^{k-1} \frac{\Delta^{j} f(a)}{\Gamma(v-k+j+1)}(t-a)^{(v-k+j)}, \\
\text { for } t \geq a-[-v] .
\end{array}
$$

Theorem 48. Let $f$ be $\Delta$-differentiable and let its highest order derivative be rd-continuous over $\mathbb{T}$. When $\alpha, \beta>0, M=[\beta]+$ 1, one has the following:

(1) $\left(D_{\Delta, t_{0}}^{\beta} I_{\Delta, t_{0}}^{\alpha} f\right)(t)=\left(I_{\Delta, t_{0}}^{\alpha-\beta}\right) f(t)$,

(2) $\left(I_{\Delta, t_{0}}^{\alpha} D_{\Delta, t_{0}}^{\beta} f\right)(t)=\left(D_{\Delta, t_{0}}^{\beta-\alpha}\right) f(t)$

$$
-\sum_{k=1}^{M} h_{\alpha-k}\left(t, t_{0}\right) D_{\Delta, t_{0}}^{\beta-k} f\left(t_{0}\right)
$$

Proof. According to Theorem 42 and (24), we have

$$
\begin{aligned}
\left(D_{\Delta, t_{0}}^{\beta} I_{\Delta, t_{0}}^{\alpha} f\right)(t) & =\left(D_{\Delta}^{M} I_{\Delta, t_{0}}^{M-\beta} I_{\Delta, t_{0}}^{\alpha} f\right)(t) \\
& =D_{\Delta}^{M}\left(h_{M+\alpha-\beta-1}\left(\cdot, t_{0}\right) * g\right)(t) \\
& =\left(h_{\alpha-\beta-1}\left(\cdot, t_{0}\right) * g\right)(t) \\
& =\left(I_{\Delta, t_{0}}^{\alpha-\beta}\right) f(t) .
\end{aligned}
$$

In addition,

$$
\begin{aligned}
I_{\Delta, t_{0}}^{\alpha} D_{\Delta, t_{0}}^{\beta} f(t)= & I_{\Delta, t_{0}}^{\alpha} D_{\Delta}^{M} I_{\Delta, t_{0}}^{M-\beta} f(t) \\
= & D_{\Delta}^{M} I_{\Delta, t_{0}}^{\alpha} I_{\Delta, t_{0}}^{M-\beta} f(t) \\
& -\sum_{k=0}^{M-1} h_{\alpha-M+k}\left(t, t_{0}\right) D_{\Delta, t_{0}}^{k+\beta-M} f\left(t_{0}\right) \\
= & I_{\Delta, t_{0}}^{\alpha-\beta} f(t)-\sum_{k=1}^{M} h_{\alpha-k}\left(t, t_{0}\right) D_{\Delta, t_{0}}^{\beta-k} f\left(t_{0}\right) .
\end{aligned}
$$
[3].

As a direct corollary of Theorem 48, we get Lemma 2.5 in

Corollary 49 (see [3]). Let $R(\alpha)>0, n=[R(\alpha)]+1$, and let $f_{n-\alpha}(x)=\left(I_{a+}^{n-\alpha} f\right)(x)$. If $f(x) \in L_{1}(a, b)$ and $f_{n-\alpha}(x) \in$ $A C^{n}[a, b]$, then

$$
\left(I_{a+}^{\alpha} D_{a+}^{\alpha} f\right)(x)=f(x)-\sum_{j=1}^{n} \frac{f_{n-\alpha}^{(n-j)}(a)}{\Gamma(\alpha-j+1)}(x-a)^{\alpha-j}
$$

holds almost everywhere on $[a, b]$.

For fractional sum and difference, there is also the following theorem in [1].

It is different from Theorem 3.3 in [1], and from Theorem 48, we can get the following corollary.

Corollary 50. Let $f: \mathbb{N}_{a} \rightarrow \mathbb{R}$ be given. For any $k \in \mathbb{N}_{0}$ and $\nu, \mu>0$ with $M-1<\mu \leq M$,

$$
\Delta_{a}^{-v} \Delta_{a}^{\mu} f(t)
$$

$$
=\Delta_{a}^{\mu-v} f(t)-\sum_{j=0}^{M-1} \frac{\Delta_{a}^{j-(M-\mu)} f(a)}{\Gamma(v-k+j+1)}(t-a)^{(v-M+j)} .
$$


Next, we will give the Laplace transform of fractional integral and derivative on time scales.

Theorem 51. (1) Let $\alpha>0$ and $f: \mathbb{T} \rightarrow \mathbb{R}$ be locally $\Delta$ integrable. For $s, t \in \mathbb{T}$ with $t>t_{0}$, one has

$$
\mathscr{L}\left\{I_{\Delta, t_{0}}^{\alpha} f(t)\right\}\left(z, t_{0}\right)=\frac{1}{z^{\alpha}} \mathscr{L}\{f(t)\}\left(z, t_{0}\right) .
$$

(2) Let $\alpha>0$ and $f: \mathbb{T} \rightarrow \mathbb{R}$ be locally $\Delta$-integrable. For $t_{0}, t \in \mathbb{T}^{k^{m}}$ with $t>s$, one has

$$
\begin{aligned}
\mathscr{L} & \left\{D_{\Delta, t_{0}}^{\alpha} f(t)\right\}\left(z, t_{0}\right) \\
& =z^{\alpha} \mathscr{L}\{f(t)\}\left(z, t_{0}\right)-\sum_{j=1}^{m} z^{j-1} D_{\Delta, t_{0}}^{\alpha-j} f\left(t_{0}\right) .
\end{aligned}
$$

Proof. (1) According to Definition 28 and convolution theorem, we have

$$
\begin{aligned}
\mathscr{L} & \left\{I_{\Delta, t_{0}}^{\alpha} f(t)\right\}\left(z, t_{0}\right) \\
& =\mathscr{L}\left\{\left(h_{\alpha-1}\left(\cdot, t_{0}\right) * f\right)(t)\right\}\left(z, t_{0}\right) \\
& =\mathscr{L}\left\{h_{\alpha-1}\left(t, t_{0}\right)\right\}\left(z, t_{0}\right) \mathscr{L}\{f(t)\}\left(z, t_{0}\right) \\
& =\frac{1}{z^{\alpha}} \mathscr{L}\{f(t)\}\left(z, t_{0}\right) .
\end{aligned}
$$

(2) By Definition 31 and (26) and taking the Laplace transform of fractional integral into account, we get

$$
\begin{aligned}
\mathscr{L} & \left\{D_{\Delta, t_{0}}^{\alpha} f(t)\right\}\left(z, t_{0}\right) \\
& =\mathscr{L}\left\{D_{\Delta, t_{0}}^{m} I_{\Delta, t_{0}}^{m-\alpha} f(t)\right\}\left(z, t_{0}\right) \\
& =z^{m} \mathscr{L}\left\{I_{\Delta, t_{0}}^{m-\alpha} f(t)\right\}\left(z, t_{0}\right)-\sum_{j=0}^{m-1} z^{m-j-1} D^{j} I_{\Delta, t_{0}}^{m-\alpha} f\left(t_{0}\right) \\
& =z^{m} \frac{1}{z^{m-\alpha}} \mathscr{L}\{f(t)\}\left(z, t_{0}\right)-\sum_{j=0}^{m-1} z^{m-j-1} D_{\Delta, t_{0}}^{j-m+\alpha} f\left(t_{0}\right) \\
& =z^{\alpha} \mathscr{L}\{f(t)\}\left(z, t_{0}\right)-\sum_{j=1}^{m} z^{j-1} D_{\Delta, t_{0}}^{\alpha-j} f\left(t_{0}\right) .
\end{aligned}
$$

\section{Cauchy Type Problem with Riemann-Liouville Fractional Derivative}

In this section, we consider Cauchy type problem with Riemann-Liouville fractional derivative

$$
\begin{gathered}
D_{\Delta, t_{0}}^{\alpha} y(t)=f(t, y(t)) \quad(\alpha>0), \\
D_{\Delta, t_{0}}^{\alpha-k} y\left(t_{0}\right)=b_{k} \quad(k=1, \ldots, m=-[-\alpha]) .
\end{gathered}
$$

In the space $L_{\Delta}^{\alpha}[a, b)$ defined for $\alpha>0$ by

$$
L_{\Delta}^{\alpha}[a, b):=\left\{y \in L_{\Delta}[a, b): D_{\Delta, t_{0}}^{\alpha} \in L_{\Delta}[a, b)\right\} .
$$

Here $L_{\Delta}[a, b):=L_{\Delta, 1}[a, b)$ is the space of $\Delta$-Lebesgue summable functions in a finite interval $\widetilde{\mathbb{T}}:=[a, b)_{\mathbb{T}}$.

In the following, we prove that Cauchy type problem and nonlinear Volterra integral equation are equivalent in the sense that if $y(t) \in L_{\Delta}[a, b)$ satisfies one of these relations, then it also satisfies the other.

Theorem 52. Let $\alpha>0, m=-[-\alpha], \widetilde{\mathbb{T}}:=[a, b]_{\mathbb{T}}, t_{0}, t \in \widetilde{\mathbb{T}}^{k^{m}}$. Let $G$ be an open set in $\mathbb{R}$ and let $f: \widetilde{\mathbb{T}} \times G \rightarrow \mathbb{R}$ be a function such that $f(t, y) \in L_{\Delta}[a, b)$ for any $y \in G$. If $y(t) \in L_{\Delta}[a, b)$, then Cauchy type problem (102) and (103) is equivalent to

$$
y(t)=\sum_{k=1}^{m} h_{\alpha-k}\left(t, t_{0}\right) b_{k}+I_{\Delta, t_{0}}^{\alpha} f(t, y(t)) .
$$

Proof. First we prove the necessity. We apply $I_{\Delta, t_{0}}^{\alpha}$ to both sides of (102) and get by Theorem 48

$$
\begin{aligned}
I_{\Delta, t_{0}}^{\alpha} D_{\Delta, t_{0}}^{\alpha} y(t) & =y(t)-\sum_{k=1}^{m} h_{\alpha-k}\left(t, t_{0}\right) D_{\Delta, t_{0}}^{\alpha-k} y\left(t_{0}\right) \\
& =\left(h_{\alpha-1}\left(\cdot, t_{0}\right) * f(\cdot, \cdot)\right)(t) . \\
& =I_{\Delta, t_{0}}^{\alpha} f(t, y(t)) .
\end{aligned}
$$

Thus

$$
y(t)=\sum_{k=1}^{m} h_{\alpha-k}\left(t, t_{0}\right) b_{k}+I_{\Delta, t_{0}}^{\alpha} f(t, y(t)) .
$$

Now we prove the sufficiency. Applying the operator $D_{\Delta, t_{0}}^{\alpha}$ to both sides of (105) and by (57) and Theorem 48(1), we have

$$
\begin{aligned}
D_{\Delta, t_{0}}^{\alpha} y(t)= & \sum_{k=1}^{m} D_{\Delta, t_{0}}^{\alpha} h_{\alpha-k}\left(t, t_{0}\right) b_{k} \\
& +D_{\Delta, t_{0}}^{\alpha} I_{\Delta, t_{0}}^{\alpha} f(t, y(t)) \\
= & f(t, y(t)) .
\end{aligned}
$$

Now we show that the relations in (103) also hold. For this, we apply the operators $D_{\Delta, t_{0}}^{\alpha-k}(k=1, \ldots, m)$ to both sides of (105):

$$
\begin{aligned}
& D_{\Delta, t_{0}}^{\alpha-k} y(t) \\
& =\sum_{j=1}^{m} D_{\Delta, t_{0}}^{\alpha-k} h_{\alpha-j}\left(t, t_{0}\right) b_{j}+D_{\Delta, t_{0}}^{\alpha-k} I_{\Delta, t_{0}}^{\alpha} f(t, y(t)) \\
& =\sum_{j=1}^{m} D_{\Delta, t_{0}}^{m-k} I_{\Delta, t_{0}}^{m-\alpha} h_{\alpha-j}\left(t, t_{0}\right) b_{j}+I_{\Delta, t_{0}}^{k} f(t, y(t)) \\
& =\sum_{j=1}^{m} D_{\Delta, t_{0}}^{m-k} h_{m-j}\left(t, t_{0}\right) b_{j}+I_{\Delta, t_{0}}^{k} f(t, y(t)) .
\end{aligned}
$$

Since

$$
\begin{aligned}
D_{\Delta, t_{0}}^{m-k} h_{m-j}\left(t, t_{0}\right) & = \begin{cases}0, & k<j, \\
1, & k=j, \\
h_{k-j}\left(t, t_{0}\right), & k>j,\end{cases} \\
D_{\Delta, t_{0}}^{\alpha-k} y\left(t_{0}\right) & =b_{k} \quad(k=1, \ldots, m) .
\end{aligned}
$$


In the following, we bring in Lipschitzian-type condition:

$$
\left|f\left(t, y_{1}(t)\right)-f\left(t, y_{2}(t)\right)\right| \leq A\left|y_{1}(t)-y_{2}(t)\right|,
$$

where $A>0$ does not depend on $t \in[a, b)_{\mathbb{T}}$. We will derive a unique solution to the Cauchy problem (102)-(103).

Theorem 53. Let the condition of Theorem 52 be valid, let $f(t, y)$ satisfy the Lipschitzian condition (111), and $|f(t, y)| \leq$ $M,{ }_{\Delta} F_{\alpha, 1}\left(A, t, t_{0}\right)$ is defined on $\widetilde{\mathbb{T}}:=[a, b]_{\mathbb{T}}$, where $A$ is the Lipschitzian constant in (111). Then there exists a unique solution $y(t)$ to initial value problem (102)-(103) in the space $L_{\Delta}^{\alpha}[a, b)$.

Proof. Since the Cauchy type problem (102)-(103) and the nonlinear Volterra integral equation (105) are equivalent, we only need to prove that there exists a unique solution to (105).

We define function sequences:

$$
y_{l}(t)=y_{0}(t)+I_{\Delta, t_{0}}^{\alpha} f\left(t, y_{l-1}(t)\right) \quad(l=1,2, \ldots,),
$$

where

$$
y_{0}(t)=\sum_{k=1}^{m} h_{\alpha-k}\left(t, t_{0}\right) b_{k} .
$$

We obtain, by induction that,

$$
\left|y_{l}(t)-y_{l-1}(t)\right| \leq M A^{l-1} h_{l \alpha}\left(t, t_{0}\right) .
$$

In fact, for $l=1$, as $|f(t, y(t))| \leq M$, we have

$$
\left|y_{1}(t)-y_{0}(t)\right| \leq M I_{\Delta, t_{0}}^{\alpha} h_{0}\left(t, t_{0}\right)=M h_{\alpha}\left(t, t_{0}\right) .
$$

If

$$
\left|y_{l-1}(t)-y_{l-2}(t)\right| \leq M A^{l-2} h_{(l-1) \alpha}\left(t, t_{0}\right)
$$

then

$$
\begin{aligned}
\left|y_{l}(t)-y_{l-1}(t)\right| & \leq A\left(I_{\Delta, t_{0}}^{\alpha}\left|y_{l-1}-y_{l-2}\right|\right)(t) \\
& \leq A\left(I_{\Delta, t_{0}}^{\alpha} M A^{l-2} h_{(l-1) \alpha}\left(\cdot, t_{0}\right)\right)(t) \\
& =M A^{l-1}\left(I_{\Delta, t_{0}}^{\alpha} h_{(l-1) \alpha}\left(\cdot, t_{0}\right)\right)(t) \\
& =M A^{l-1} h_{l \alpha}\left(t, t_{0}\right) .
\end{aligned}
$$

Let

$$
y(t)=\lim _{l \rightarrow \infty}\left(y_{l}(t)-y_{0}(t)\right)=\sum_{l=1}^{\infty}\left(y_{l}(t)-y_{l-1}(t)\right),
$$

and we have

$$
\begin{aligned}
\sum_{l=1}^{\infty}\left|y_{l}(t)-y_{l-1}(t)\right| & \leq \sum_{l=1}^{\infty} M A^{l-1} h_{l \alpha}\left(t, t_{0}\right) \\
& =\frac{M}{A} \sum_{l=1}^{\infty} A^{l} h_{l \alpha}\left(t, t_{0}\right) \\
& <\frac{M}{A} \sum_{l=1}^{\infty} A^{l} h_{l \alpha}\left(b, t_{0}\right) \\
& =\frac{M}{A}\left[{ }_{\Delta} F_{\alpha, 1}\left(A, b, t_{0}\right)-1\right] .
\end{aligned}
$$

By Weierstrass discriminance, we obtain $y_{l}(t)$ convergent uniformly. Next we will show the uniqueness. Assume that $z(t)$ is another solution to (105); that is,

$$
z(t)=y_{0}(t)+I_{\Delta, t_{0}}^{\alpha} f(t, z(t)) .
$$

As

$$
\begin{gathered}
|f(t, y(t))| \leq M \\
\left|y_{0}(t)-z(t)\right| \leq I_{\Delta, t_{0}}^{\alpha}|f(t, z(t))| \leq M h_{\alpha}\left(t, t_{0}\right) .
\end{gathered}
$$

If

$$
\left|y_{l-1}(t)-z(t)\right| \leq M A^{l-1} h_{l \alpha}\left(t, t_{0}\right)
$$

then

$$
\begin{aligned}
\left|y_{l}(t)-z(t)\right| & \leq\left(I_{\Delta, t_{0}}^{\alpha}\left|f\left(\cdot, y_{l-1}(\cdot)\right)-f(\cdot, z(\cdot))\right|\right)(t) \\
& \leq\left(I_{\Delta, t_{0}}^{\alpha} A\left|y_{l-1}-z\right|\right)(t) \\
& \leq\left(I_{\Delta, t_{0}}^{\alpha} A M A^{l-1} h_{l \alpha}\left(\cdot, t_{0}\right)\right)(t) \\
& \leq M A^{l}\left(I_{\Delta, t_{0}}^{\alpha} h_{l \alpha}\left(\cdot, t_{0}\right)\right)(t) \\
& =M A^{l} h_{(l+1) \alpha}\left(t, t_{0}\right) .
\end{aligned}
$$

By mathematical induction, we have

$$
\left|y_{l}(t)-z(t)\right| \leq M A^{l} h_{(l+1) \alpha}\left(t, t_{0}\right)
$$

and then get $z(t)=y(t)$ owing to the uniqueness of the limit. This completes the proof of the theorem.

Next we consider the generalized Cauchy type problem:

$$
\begin{array}{r}
D_{\Delta, t_{0}}^{\alpha} y(t)=f\left(t, y(t), D_{\Delta, t_{0}}^{\alpha_{1}} y(t), \ldots, D_{\Delta, t_{0}}^{\alpha_{l}} y(t)\right) \\
\left(0=\alpha_{0} \leq \alpha_{1} \leq \cdots \leq \alpha_{l}<\alpha\right), \\
D_{\Delta, t_{0}}^{\alpha-k} y\left(t_{0}\right)=b_{k} \quad(k=1, \ldots, n, n=[\alpha]+1) .
\end{array}
$$

Theorem 54. Let $f:[a, b)_{\mathbb{T}} \times G \rightarrow \mathbb{R}$ be a function such that $f\left(t, y, y_{1}, \ldots, y_{l}\right) \in L_{\Delta}[a, b)$ for any $\left(y, y_{1}, \ldots, y_{l}\right) \in G$. If $y(t) \in L_{\Delta}[a, b)$, then $y(t)$ satisfies a.e. the relations (126) and (125) if and only if it satisfies a.e. the integral equation

$$
\begin{aligned}
y(t)= & \sum_{k=1}^{n} h_{\alpha-k}\left(t, t_{0}\right) b_{k} \\
& +\left(I_{\Delta, t_{0}}^{\alpha} f\left(\tau, y(\tau), D_{\Delta, t_{0}}^{\alpha_{1}} y(\tau), \ldots, D_{\Delta, t_{0}}^{\alpha_{l}} y(\tau)\right)\right)(t) .
\end{aligned}
$$

Assume that $f$ satisfies generalized Lipschitzian condition

$$
\left|f\left(t, y, y_{1}, \ldots, y_{l}\right)-f\left(t, z, z_{1}, \ldots, z_{l}\right)\right| \leq A\left[\sum_{j=1}^{l}\left|y_{j}-z_{j}\right|\right] .
$$

According to Theorem 54 and by a similar proof to that of Theorem 53, we have the following theorem. 
Theorem 55. Let the condition of Theorem 54 be valid and let $f\left(t, y, y_{1}, \ldots, y_{l}\right)$ satisfy the Lipschitzian condition (128). Then there exists a unique solution $y(t)$ to the generalized Cauchy type problem.

\section{The Dependency of the Solution upon the Initial Value}

We consider fractional differential initial value problem again:

$$
\begin{gathered}
D_{\Delta, t_{0}}^{\alpha} y(t)=f(t, y(t)), \\
D_{\Delta, t_{0}}^{\alpha} y\left(t_{0}\right)=\eta,
\end{gathered}
$$

where $0<\alpha<1$.

Using Theorem 52, we have

$$
y(t)=\eta h_{\alpha-1}\left(t, t_{0}\right)+\left(I_{\Delta, t_{0}}^{\alpha} f(\tau, y(\tau))\right)(t) .
$$

Suppose that $z(t)$ is the solution to the initial value problem:

$$
\begin{gathered}
D_{\Delta, t_{0}}^{\alpha} y(t)=f(t, y(t)), \\
D_{\Delta, t_{0}}^{\alpha} y\left(t_{0}\right)=\bar{\eta} .
\end{gathered}
$$

We can derive the dependency of the solution upon the initial value.

Theorem 56. Let $\widetilde{\mathbb{T}}:=[a, b]_{\mathbb{T}}, t_{0}, t \in \widetilde{\mathbb{T}}^{k}$ and suppose that $f$ satisfy the Lipschitz condition; that is,

$$
|f(t, z(t))-f(t, y(t))| \leq A|z(t)-y(t)| .
$$

Then one has

$$
|z(t)-y(t)| \leq|\bar{\eta}-\eta|_{\Delta} F_{\alpha, \alpha}\left(A, b, t_{0}\right)
$$

Proof. By the proof of Theorem 53, we know that $y(t)=$ $\lim _{m \rightarrow \infty} y_{m}(t), z(t)=\lim _{m \rightarrow \infty} z_{m}(t)$, where

$$
\begin{gathered}
y_{0}(t)=\eta h_{\alpha-1}\left(t, t_{0}\right), \\
y_{m}(t)=y_{0}(t)+\left(I_{\Delta, t_{0}}^{\alpha} f\left(\tau, y_{m-1}(\tau)\right)\right)(t), \\
z_{0}(t)=\bar{\eta} h_{\alpha-1}\left(t, t_{0}\right), \\
z_{m}(t)=z_{0}(t)+\left(I_{\Delta, t_{0}}^{\alpha} f\left(\tau, z_{m-1}(\tau)\right)\right)(t) .
\end{gathered}
$$

Using the Lipschitz condition, we have

$$
\begin{aligned}
\mid z_{1}(t) & -y_{1}(t) \mid \\
\leq & |\bar{\eta}-\eta| h_{\alpha-1}\left(t, t_{0}\right) \\
& +\left(I_{\Delta, t_{0}}^{\alpha}\left|f\left(\tau, z_{0}(\tau)\right)-f\left(\tau, y_{0}(\tau)\right)\right|\right)(t) \\
\leq & |\bar{\eta}-\eta| h_{\alpha-1}\left(t, t_{0}\right)+\left(I_{\Delta, t_{0}}^{\alpha} A\left|z_{0}(\tau)-y_{0}(\tau)\right|\right)(t) \\
= & |\bar{\eta}-\eta| h_{\alpha-1}\left(t, t_{0}\right)+|\bar{\eta}-\eta| A\left(I_{\Delta, t_{0}}^{\alpha} h_{\alpha-1}\left(\tau, t_{0}\right)\right)(t) \\
= & |\bar{\eta}-\eta| h_{\alpha-1}\left(t, t_{0}\right)+|\bar{\eta}-\eta| A h_{2 \alpha-1}\left(t, t_{0}\right) \\
= & |\bar{\eta}-\eta|\left[h_{\alpha-1}\left(t, t_{0}\right)+A h_{2 \alpha-1}\left(t, t_{0}\right)\right] .
\end{aligned}
$$

Suppose that

$$
\begin{aligned}
& \left|z_{m-1}(t)-y_{m-1}(t)\right| \\
& \quad \leq|\bar{\eta}-\eta| \sum_{j=0}^{m-1} A^{j} h_{j \alpha+\alpha-1}\left(t, t_{0}\right) .
\end{aligned}
$$

Then

$$
\begin{aligned}
\mid z_{m}( & t)-y_{m}(t) \mid \\
\leq & |\bar{\eta}-\eta| h_{\alpha-1}\left(t, t_{0}\right) \\
& +\left(I_{\Delta, t_{0}}^{\alpha}\left|f\left(\tau, z_{m-1}(\tau)\right)-f\left(\tau, y_{m-1}(\tau)\right)\right|\right)(t) \\
\leq & |\bar{\eta}-\eta| h_{\alpha-1}\left(t, t_{0}\right) \\
& +\left(I_{\Delta, t_{0}}^{\alpha} A\left|z_{m-1}(\tau)-y_{m-1}(\tau)\right|\right)(t) \\
\leq & |\bar{\eta}-\eta| h_{\alpha-1}\left(t, t_{0}\right) \\
& +\left(I_{\Delta, t_{0}}^{\alpha} A|\bar{\eta}-\eta| \sum_{j=0}^{m-1} A^{j} h_{j \alpha+\alpha-1}\left(\tau, t_{0}\right)\right)(t) \\
= & |\bar{\eta}-\eta| h_{\alpha-1}\left(t, t_{0}\right) \\
& +A|\bar{\eta}-\eta| \sum_{j=0}^{m-1} A^{j}\left(I_{\Delta, t_{0}}^{\alpha} h_{j \alpha+\alpha-1}\left(\tau, t_{0}\right)\right)(t) \\
= & |\bar{\eta}-\eta| h_{\alpha-1}\left(t, t_{0}\right) \\
& +|\bar{\eta}-\eta| \sum_{j=0}^{m-1} A^{j+1} h_{(j+1) \alpha+\alpha-1}\left(t, t_{0}\right) \\
= & |\bar{\eta}-\eta| \sum_{j=0}^{m} A^{j} h_{j \alpha+\alpha-1}\left(t, t_{0}\right) . \\
& \\
&
\end{aligned}
$$

According to mathematical induction, we have

$$
\left|z_{m}(t)-y_{m}(t)\right| \leq|\bar{\eta}-\eta| \sum_{j=0}^{m} A^{j} h_{j \alpha+\alpha-1}\left(t, t_{0}\right) .
$$

Taking the limit $m \rightarrow \infty$, we obtain that

$$
\begin{aligned}
|z(t)-y(t)| & \leq|\bar{\eta}-\eta| \sum_{j=0}^{\infty} A^{j} h_{j \alpha+\alpha-1}\left(t, t_{0}\right) \\
& \leq|\bar{\eta}-\eta|_{\Delta} F_{\alpha, \alpha}\left(A, b, t_{0}\right) .
\end{aligned}
$$

As a special case, when fractional equation is linear, we can obtain its explicit solutions and we will explain it in next section. 


\section{Homogeneous Equations with Constant Coefficients}

In this section, we apply the Laplace transform method to derive explicit solutions to homogeneous equations of the form

$$
\begin{gathered}
\sum_{k=1}^{m} A_{k}\left[D_{\Delta, t_{0}}^{\alpha_{k}} y(t)\right]+A_{0} y(t)=0 \\
\left(t>t_{0} ; m \in \mathbb{N} ; 0<\alpha_{1}<\cdots<\alpha_{m}\right)
\end{gathered}
$$

with the Liouville fractional derivatives $D_{\Delta, t_{0}}^{\alpha_{k}} y(k=1, \ldots$, $m)$. Here $A_{k} \in \mathbb{R}(k=0, \ldots, m)$ are real constants, and, generally speaking, we can take $A_{m}=1$.

In order to solve the equation, we need the following Laplace transform formula:

$$
\begin{gathered}
\mathscr{L}\left\{D_{\Delta, t_{0}}^{\alpha} y(t)\right\}\left(z, t_{0}\right)=z^{\alpha} \mathscr{L}\{y(t)\}\left(z, t_{0}\right) \\
-\sum_{j=1}^{l} d_{j} z^{j-1} \quad(l-1<\alpha \leq l \in \mathbb{N}), \\
d_{j}=D_{\Delta, t_{0}}^{\alpha-j} y\left(t_{0}\right) \quad(j=1, \ldots, l) .
\end{gathered}
$$

First, we derive explicit solutions to (140) with $m=1$ :

$$
D_{\Delta, t_{0}}^{\alpha} y(t)-\lambda y(t)=0 \quad\left(t>t_{0} ; l-1<\alpha \leq l ; l \in \mathbb{N} ; \lambda \in \mathbb{R}\right) .
$$

In order to prove our result, we need the following definition and lemma.

Definition 57. The function $W_{\alpha}(t)$ is defined by

$$
W_{\alpha}(t)=\operatorname{det}\left(\left(D_{\Delta, t_{0}}^{\alpha-k} y_{j}\right)(t)\right)_{k, j=1}^{n} \quad(n=[\alpha]+1, a \leq t \leq b) .
$$

Lemma 58. The solutions $y_{1}(t), y_{2}(t), \ldots, y_{n}(t)$ are linearly independent if and only if $W_{\alpha}\left(t^{*}\right) \neq 0$ at some point $t^{*} \in[a, b]$.

Proof. We first prove sufficiency. If, to the contrary, $y_{j}(t)(j=$ $1,2, \ldots, n)$ are linearly dependent on $\Omega$, then there exist $n$ constants $\left\{c_{j}\right\}_{j=1}^{n}$, not all zero, such that

$$
\left(\left(D_{\Delta, t_{0}}^{\alpha-k} y_{j}\right)(t)\right)_{k, j=1}^{n}\left(\begin{array}{c}
c_{1} \\
c_{2} \\
\vdots \\
c_{n}
\end{array}\right) \equiv 0
$$

holds, and thus $W_{\alpha}(t) \equiv 0$ which leads to a contradiction. Therefore, if $W_{\alpha}\left(t^{*}\right) \neq 0$ at some point $t^{*} \in \Omega$, then $y_{1}(t), y_{2}(t), \ldots, y_{n}(t)$ are linearly independent. Now we prove the necessity. Suppose, to the contrary, for $t \in \Omega, W_{\alpha}(t)=0$. Consider

$$
\left(\left(D_{\Delta, t_{0}}^{\alpha-k} y_{j}\right)\left(t^{*}\right)\right)_{k, j=1}^{n} C=0
$$

where $t^{*} \in \Omega, C=\left(\begin{array}{c}c_{1} \\ c_{2} \\ \vdots \\ c_{n}\end{array}\right)$. As $W_{\alpha}\left(t^{*}\right)=0$, the equations have nontrivial solution $c_{j}(j=1,2, \ldots, n)$. Now we construct a function using these constants:

$$
y(t)=\sum_{j=1}^{n} c_{j} y_{j}(t)
$$

and we get that $y(t)$ is a solution. From (146), we obtain that $y(t)$ satisfies initial value condition

$$
\left(D_{\Delta, t_{0}}^{\alpha-k} y\left(t^{*}\right)\right)=0, \quad k=1, \ldots, n .
$$

However, $y(t)=0$ is also a solution to equation satisfying the initial value condition. By the uniqueness of solution, we have

$$
\sum_{j=1}^{n} c_{j} y_{j}(t)=0
$$

and, thus $y_{j}(t)(j=1,2, \ldots, n)$ are linearly dependant, which leads to a contradiction. Thus, if the solutions $y_{1}(t)$, $y_{2}(t), \ldots, y_{n}(t)$ are linearly independent, then $W_{\alpha}\left(t^{*}\right) \neq 0$ at some point $t^{*} \in \Omega$.

There hold the following statements.

Theorem 59. Let $l-1<\alpha \leq l(l \in \mathbb{N})$ and $\lambda \in \mathbb{R}$. Then the functions

$$
\begin{array}{r}
y_{j}(t)={ }_{\Delta} F_{\alpha, \alpha+1-j}\left(\lambda, t, t_{0}\right)\left(=\sum_{k=0}^{\infty} \lambda^{k} \widehat{h}_{k \alpha+\alpha-j}\left(t, t_{0}\right)\right) \\
(j=1, \ldots, l)
\end{array}
$$

yield the fundamental system of solutions to (143). Moreover, $y_{j}(t), j=1,2, \ldots, l$, satisfy

$$
\begin{gathered}
D_{\Delta, t_{0}}^{\alpha-k} y_{j}\left(t_{0}\right)=0 \quad(k, j=1, \ldots, l ; k \neq j), \\
D_{\Delta, t_{0}}^{\alpha-k} y_{k}\left(t_{0}\right)=1 \quad(k=1, \ldots, l) .
\end{gathered}
$$

Proof. Applying the Laplace transform to (143) and taking (141) into account, we have

$$
\mathscr{L}\{y(t)\}\left(z, t_{0}\right)=\sum_{j=1}^{l} d_{j} \frac{z^{j-1}}{z^{\alpha}-\lambda},
$$

where $d_{j}(j=1, \ldots, l)$ are given by $(142)$.

Formula (49) with $\beta=\alpha+1-j$ yields

$$
\mathscr{L}\left\{{ }_{\Delta} F_{\alpha, \alpha+1-j}\left(\lambda, t, t_{0}\right)\right\}\left(z, t_{0}\right)=\frac{z^{j-1}}{z^{\alpha}-\lambda} \quad\left(|\lambda|<|z|^{\alpha}\right) .
$$

Thus, from (152), we derive the following solution to (143):

$$
y(t)=\sum_{j=1}^{l} d_{j} y_{j}(t), \quad y_{j}(t)={ }_{\Delta} F_{\alpha, \alpha+1-j}\left(\lambda, t, t_{0}\right) .
$$


It is easily verified that the functions $y_{j}(t)$ are solutions to (143):

$$
\begin{aligned}
& D_{\Delta, t_{0}}^{\alpha}\left[{ }_{\Delta} F_{\alpha, \alpha+1-j}\left(\lambda, t, t_{0}\right)\right] \\
& \quad=\lambda_{\Delta} F_{\alpha, \alpha+1-j}\left(\lambda, t, t_{0}\right) \quad(j=1, \ldots, l) .
\end{aligned}
$$

In fact,

$$
\begin{aligned}
D_{\Delta, t_{0}}^{\alpha}\left[{ }_{\Delta} F_{\alpha, \alpha+1-j}\left(\lambda, t, t_{0}\right)\right] & =D_{\Delta, t_{0}}^{\alpha}\left[\sum_{k=0}^{\infty} \lambda^{k} h_{k \alpha+\alpha-j}\left(t, t_{0}\right)\right] \\
& =\sum_{k=0}^{\infty} \lambda^{k} h_{k \alpha-j}\left(t, t_{0}\right) \\
& =\sum_{k=-1}^{\infty} \lambda^{k+1} h_{(k+1) \alpha-j}\left(t, t_{0}\right) \\
& =\lambda^{0} h_{-j}\left(t, t_{0}\right) \\
& +\sum_{k=0}^{\infty} \lambda^{k+1} h_{(k+1) \alpha-j}\left(t, t_{0}\right) \\
= & \lambda \sum_{k=0}^{\infty} \lambda^{k} h_{k \alpha+\alpha-j}\left(t, t_{0}\right) \\
= & \lambda F_{\Delta, \alpha+1-j}\left(\lambda, t, t_{0}\right) .
\end{aligned}
$$

Moreover,

$$
\begin{aligned}
D_{\Delta, t_{0}}^{\alpha-k} y_{j}(t) & =D_{\Delta, t_{0}}^{\alpha-k}\left[\sum_{s=0}^{\infty} \lambda^{s} \widehat{h}_{s \alpha+\alpha-j}\left(t, t_{0}\right)\right] \\
& =\sum_{s=0}^{\infty} \lambda^{s} h_{s \alpha+k-j}\left(t, t_{0}\right) .
\end{aligned}
$$

It follows from (157) that

$$
\begin{gathered}
D_{\Delta, t_{0}}^{\alpha-k} y_{j}\left(t_{0}\right)=0 \quad(k, j=1, \ldots, l ; k>j), \\
D_{\Delta, t_{0}}^{\alpha-k} y_{k}\left(t_{0}\right)=1 \quad(k=1, \ldots, l) .
\end{gathered}
$$

If $k<j$, then

$$
\begin{aligned}
D_{\Delta, t_{0}}^{\alpha-k} y_{j}(t) & =\sum_{s=1}^{\infty} \lambda^{s} h_{s \alpha+k-j}\left(t, t_{0}\right) \\
& =\sum_{s=0}^{\infty} \lambda^{s+1} h_{s \alpha+\alpha+k-j}\left(t, t_{0}\right),
\end{aligned}
$$

and since $\alpha+k-j \geq \alpha+1-l>0$ for any $k, j=1, \ldots, l$, the following relations hold:

$$
D_{\Delta, t_{0}}^{\alpha-k} y_{j}\left(t_{0}\right)=0 \quad(k, j=1, \ldots, l ; k<j) .
$$

By (158) and (160), $W_{\alpha}\left(t_{0}\right)=1$. Then $y_{j}(t), j=1, \ldots, l$, yield the fundamental system of solutions to (143).
Corollary 60. Consider that

$$
D_{\Delta, t_{0}}^{\alpha} y(t)-\lambda y(t)=0 \quad\left(t>t_{0} ; 0<\alpha \leq 1 ; \lambda \in \mathbb{R}\right)
$$

has its solution given by

$$
y(t)={ }_{\Delta} F_{\alpha, \alpha}\left(\lambda, t, t_{0}\right),
$$

while

$$
D_{\Delta, t_{0}}^{\alpha} y(t)-\lambda y(t)=0 \quad\left(t>t_{0} ; 1<\alpha \leq 2 ; \lambda \in \mathbb{R}\right)
$$

has the fundamental system of solutions given by

$$
y_{1}(t)={ }_{\Delta} F_{\alpha, \alpha}\left(\lambda, t, t_{0}\right), \quad y_{2}(t)={ }_{\Delta} F_{\alpha, \alpha-1}\left(\lambda, t, t_{0}\right) .
$$

Next we derive the explicit solutions to (140) with $m=2$ of the form

$$
\begin{array}{r}
D_{\Delta, t_{0}}^{\alpha} y(t)-\lambda D_{\Delta, t_{0}}^{\beta} y(t)-\mu y(t)=0 \\
\left(t>t_{0} ; l-1<\alpha \leq l ; l \in \mathbb{N} ; 0<\beta<\alpha\right)
\end{array}
$$

with $\lambda, \mu \in \mathbb{R}$.

Theorem 61. Let $l-1<\alpha \leq l(l \in \mathbb{N}), 0<\beta<\alpha$, and $\lambda, \mu \in \mathbb{R}$. Then the functions

$$
y_{j}(t)=\sum_{k=0}^{\infty} \frac{\mu^{k}}{k !} \frac{\partial^{k}}{\partial \lambda^{k}}{ }_{\Delta} F_{\alpha-\beta, \alpha+k \beta+1-j}\left(\lambda, t, t_{0}\right)
$$

yield the fundamental system of solutions to (165) provided that the series in (166) are convergent. Moreover, if $\alpha+1-l>\beta>$ $l-1$, then $y_{j}(t), j=1,2, \ldots, l$, in (166) satisfy (151).

Proof. Let $m-1<\beta \leq m(m \leq l ; m \in \mathbb{N})$. Applying the Laplace transform to (165) and using (141) as in (152), we obtain

$$
\mathscr{L}\{y(t)\}\left(z, t_{0}\right)=\sum_{j=1}^{l} d_{j} \frac{z^{j-1}}{z^{\alpha}-\lambda z^{\beta}-\mu},
$$

where $d_{j}=D_{\Delta, t_{0}}^{\alpha-j} y\left(t_{0}\right)-\lambda D_{\Delta, t_{0}}^{\beta-j} y\left(t_{0}\right)(j=1, \ldots, m), d_{j}=$ $D_{\Delta, t_{0}}^{\alpha-j} y\left(t_{0}\right)(j=m+1, \ldots, l)$.

For $z \in \mathbb{C}$ and $\left|\mu z^{-\beta} /\left(z^{\alpha-\beta}-\lambda\right)\right|<1$, we have

$$
\begin{aligned}
\frac{1}{z^{\alpha}-\lambda z^{\beta}-\mu} & =\frac{z^{-\beta}}{z^{\alpha-\beta}-\lambda} \cdot \frac{1}{1-\mu z^{-\beta} /\left(z^{\alpha-\beta}-\lambda\right)} \\
& =\sum_{k=0}^{\infty} \frac{\mu^{k} z^{-\beta-k \beta}}{\left(z^{\alpha-\beta}-\lambda\right)^{k+1}},
\end{aligned}
$$

and hence (167) has the following representation:

$$
\mathscr{L}\{y(t)\}\left(z, t_{0}\right)=\sum_{j=1}^{l} d_{j} \sum_{k=0}^{\infty} \mu^{k} \frac{z^{j-1-\beta-k \beta}}{\left(z^{\alpha-\beta}-\lambda\right)^{k+1}} .
$$


By (51), for $z \in \mathbb{C}$ and $\left|\lambda z^{\beta-\alpha}\right|<1$, we have

$$
\begin{aligned}
\frac{z^{j-1-\beta-k \beta}}{\left(z^{\alpha-\beta}-\lambda\right)^{k+1}} & =\frac{z^{(\alpha-\beta)-(\alpha+k \beta+1-j)}}{\left(z^{\alpha-\beta}-\lambda\right)^{k+1}} \\
& =\frac{1}{k !} \mathscr{L}\left\{\frac{\partial^{k}}{\partial \lambda^{k}}{ }_{\Delta} F_{\alpha-\beta, \alpha+k \beta+1-j}\left(\lambda, t, t_{0}\right)\right\}\left(z, t_{0}\right) .
\end{aligned}
$$

From (169) and (170), we derive the solution to (165) as

$$
y(t)=\sum_{j=1}^{l} d_{j} y_{j}(t)
$$

where $y_{j}(t)(j=1, \ldots, l)$ are given by (166). For $q, j=$ $1, \ldots, l$, the direct evaluation yields

$$
\begin{aligned}
& D_{\Delta, t_{0}}^{\alpha-q} y_{j}(t) \\
& =D_{\Delta, t_{0}}^{\alpha-q} \sum_{k=0}^{\infty} \frac{\mu^{k}}{k !} \frac{\partial^{k}}{\partial \lambda^{k}}{ }_{\Delta} F_{\alpha-\beta, \alpha+k \beta+1-j}\left(\lambda, t, t_{0}\right) \\
& =\sum_{k=0}^{\infty} \frac{\mu^{k}}{k !} \frac{\partial^{k}}{\partial \lambda^{k}} D_{\Delta, t_{0}}^{\alpha-q}\left[\sum_{s=0}^{\infty} \lambda^{s} \widehat{h}_{s(\alpha-\beta)+\alpha+k \beta-j}\left(t, t_{0}\right)\right] \\
& =\sum_{k=0}^{\infty} \frac{\mu^{k}}{k !} \frac{\partial^{k}}{\partial \lambda^{k}} \sum_{s=0}^{\infty} \lambda^{s} \widehat{h}_{s(\alpha-\beta)+k \beta+q-j}\left(t, t_{0}\right) .
\end{aligned}
$$

For $q>j, D_{\Delta, t_{0}}^{\alpha-q} y_{j}\left(t_{0}\right)=0$, and for $q=j, D_{\Delta, t_{0}}^{\alpha-q} y_{j}\left(t_{0}\right)=$ 1. Thus we have $W_{\alpha}\left(t_{0}\right)=1$. It follows from Lemma 58 that the functions $y_{j}(t), j=1,2, \ldots, l$ in (166) are linearly independent solutions, and then they yield the fundamental system of solutions to (165). Furthermore, if $q<j$, then we rewrite (172) as follows:

$$
\begin{aligned}
D_{\Delta, t_{0}}^{\alpha-q} y_{j}(t)= & D_{\Delta, t_{0}}^{\alpha-q} \widehat{h}_{\alpha-j}\left(t, t_{0}\right) \\
& +\sum_{s=1}^{\infty} \lambda^{s} \widehat{h}_{s(\alpha-\beta)+q-j}\left(t, t_{0}\right) \\
& +\sum_{k=1}^{\infty} \frac{\mu^{k}}{k !} \frac{\partial^{k}}{\partial \lambda^{k}} \widehat{h}_{k \beta+q-j}\left(t, t_{0}\right) \\
& +\sum_{k=1}^{\infty} \frac{\mu^{k}}{k !} \frac{\partial^{k}}{\partial \lambda^{k}} \sum_{s=1}^{\infty} \lambda^{s} \widehat{h}_{s(\alpha-\beta)+k \beta+q-j}\left(t, t_{0}\right) \\
= & \sum_{s=1}^{\infty} \lambda^{s} \widehat{h}_{s(\alpha-\beta)+q-j}\left(t, t_{0}\right) \\
& +\sum_{k=1}^{\infty} \frac{\mu^{k}}{k !} \frac{\partial^{k}}{\partial \lambda^{k}} \widehat{h}_{k \beta+q-j}\left(t, t_{0}\right) \\
& +\sum_{k=1}^{\infty} \frac{\mu^{k}}{k !} \frac{\partial^{k}}{\partial \lambda^{k}} \sum_{s=1}^{\infty} \lambda^{s} \widehat{h}_{s(\alpha-\beta)+k \beta+q-j}\left(t, t_{0}\right) .
\end{aligned}
$$

If $\alpha+1-l>\beta>l-1$, then $s(\alpha-\beta)+q-j \geq(\alpha-\beta)+1-l>0$ for $k=0, q, j=1, \ldots, l, s \in \mathbb{N}^{+}$, and $k \beta+q-j \geq \beta+1-l>0$ for $s=0, q, j=1, \ldots, l, k \in \mathbb{N}^{+}$. Besides, we also have $s(\alpha-$ $\beta)+k \beta+q-j \geq(\alpha-\beta)+\beta+1-l=\alpha+1-l>0$ for $q, j=1, \ldots, l, s, k \in \mathbb{N}^{+}$. These imply that $D_{\Delta, t_{0}}^{\alpha-q} y_{j}\left(t_{0}\right)=0$. Thus the relations in (151) are valid. The proof is finished.

Corollary 62. Consider that

$$
\begin{array}{r}
D_{\Delta, t_{0}}^{\alpha} y(t)-\lambda D_{\Delta, t_{0}}^{\beta} y(t)=0 \\
\left(t>t_{0} ; l-1<\alpha \leq l ; l \in \mathbb{N} ; 0<\beta<\alpha\right)
\end{array}
$$

has its fundamental system of solution given by

$$
y_{j}(t)={ }_{\Delta} F_{\alpha-\beta, \alpha+1-j}\left(\lambda, t, t_{0}\right) \quad(j=1, \ldots, l) .
$$

Finally, we find the explicit solutions to (140) with any $m \in$ $\mathbb{N} \backslash\{1,2\}$. It is convenient to rewrite (140) in the form

$$
\begin{aligned}
& D_{\Delta, t_{0}}^{\alpha} y(t)-\lambda D_{\Delta, t_{0}}^{\beta} y(t)-\sum_{k=0}^{m-2} A_{k} D_{\Delta, t_{0}}^{\alpha_{k}} y(t)=0 \\
& \left(t>t_{0} ; m \in \mathbb{N} \backslash\{1,2\} ; 0=\alpha_{0}<\alpha_{1}\right. \\
& \left.\quad<\cdots<\alpha_{m-2}<\beta<\alpha ; \lambda, A_{0}, \ldots, A_{m-2} \in \mathbb{R}\right) .
\end{aligned}
$$

Theorem 63. Let $m \in \mathbb{N} \backslash\{1,2\}, l-1<\alpha \leq l(l \in \mathbb{N})$, let $\beta$ and $\alpha_{1}, \ldots, \alpha_{m-2}$ be such that $\alpha>\beta>\alpha_{m-2}>\cdots>\alpha_{1}>\alpha_{0}=0$, and let $\lambda, A_{0}, \ldots, A_{m-2} \in \mathbb{R}$. Then the functions

$$
\begin{aligned}
y_{j}(t)= & \sum_{n=0}^{\infty}\left(\sum_{k_{0}+\cdots+k_{m-2}=n}\right) \frac{1}{k_{0} ! \cdots k_{m-2} !} \\
& \times\left[\prod_{\nu=0}^{m-2}\left(A_{\nu}\right)^{k_{\nu}}\right] \frac{\partial^{n}}{\partial \lambda^{n}} F_{\alpha-\beta, \alpha+1-j+\sum_{\nu=0}^{m-2}\left(\beta-\alpha_{\nu}\right) k_{\nu}}\left(\lambda, t, t_{0}\right)
\end{aligned}
$$

yield the fundamental system of solutions to (176) provided that the series in (177) are convergent. The inner sum is taken over all $k_{0}, \ldots, k_{m-2} \in \mathbb{N}_{0}$ such that $k_{0}+\cdots+k_{m-2}=n$. Moreover, if $\alpha+1-l>\beta>\alpha_{m-2}+l-1$, then $y_{j}(t), j=1,2, \ldots, l$, in (177) satisfy (151).

Proof. Let $l_{m-1}-1<\beta \leq l_{m-1}, l_{k}-1<\alpha_{k}<l_{k}(k=1, \ldots, m-$ $\left.2 ; 0 \leq l_{1} \leq \cdots \leq l_{m-1} \leq l\right)$. Applying the Laplace transform to (176) and using (141) as in (167), we obtain

$$
\mathscr{L}\{y(t)\}\left(z, t_{0}\right)=\sum_{j=1}^{l} d_{j} \frac{z^{j-1}}{z^{\alpha}-\lambda z^{\beta}-\sum_{k=0}^{m-2} A_{k} z^{\alpha_{k}}},
$$


where

$$
\begin{aligned}
d_{j}= & D_{\Delta, t_{0}}^{\alpha-j} y\left(t_{0}\right)-\lambda D_{\Delta, t_{0}}^{\beta-j} y\left(t_{0}\right) \\
& -\sum_{k=1}^{m-2} A_{k} D_{\Delta, t_{0}}^{\alpha_{k}-j} y\left(t_{0}\right) \quad\left(j=1, \ldots, l_{1}\right), \\
d_{j}= & D_{\Delta, t_{0}}^{\alpha-j} y\left(t_{0}\right)-\lambda D_{\Delta, t_{0}}^{\beta-j} y\left(t_{0}\right) \\
& -\sum_{k=2}^{m-2} A_{k} D_{\Delta, t_{0}}^{\alpha_{k}-j} y\left(t_{0}\right) \quad\left(j=l_{1}+1 \ldots, l_{2}\right), \\
& \vdots \\
d_{j}= & D_{\Delta, t_{0}}^{\alpha-j} y\left(t_{0}\right)-\lambda D_{\Delta, t_{0}}^{\beta-j} y\left(t_{0}\right) \quad\left(j=l_{m-2}+1, \ldots, l_{m-1}\right), \\
d_{j}= & D_{\Delta, t_{0}}^{\alpha-j} y\left(t_{0}\right) \quad\left(j=l_{m-1}+1, \ldots, l\right) .
\end{aligned}
$$

Here $\sum_{k=m}^{n} A_{k}:=0(m>n)$. For $z \in \mathbb{C}$ and $\left|\sum_{k=0}^{m-2} A_{k} z^{\alpha_{k}-\beta} /\left(z^{\alpha-\beta}-\lambda\right)\right|<1$, we have

$$
\begin{aligned}
\frac{1}{z^{\alpha}-} & \lambda z^{\beta}-\sum_{k=0}^{m-2} A_{k} z^{\alpha_{k}} \\
= & \frac{z^{-\beta}}{z^{\alpha-\beta}-\lambda} \cdot \frac{1}{\left(1-\sum_{k=0}^{m-2} A_{k} z^{\alpha_{k}-\beta} /\left(z^{\alpha-\beta}-\lambda\right)\right)} \\
= & \sum_{n=0}^{\infty} \frac{z^{-\beta}}{\left(z^{\alpha-\beta}-\lambda\right)^{n+1}}\left(\sum_{k=0}^{m-2} A_{k} z^{\alpha_{k}-\beta}\right)^{n} \\
= & \sum_{n=0}^{\infty}\left(\sum_{k_{0}+\cdots+k_{m-2}=n}\right) \frac{n !}{k_{0} ! \cdots k_{m-2} !} \\
& \times\left[\prod_{\nu=0}^{m-2}\left(A_{\nu}\right)^{k_{\nu}}\right] \frac{z^{-\beta-\sum_{v=0}^{m-2}\left(\beta-\alpha_{\nu}\right) k_{\nu}}}{\left(z^{\alpha-\beta}-\lambda\right)^{n+1}},
\end{aligned}
$$

if we also take into account the following relation:

$$
\begin{aligned}
& \left(x_{0}+\cdots+x_{m-2}\right)^{n} \\
& =\left(\sum_{k_{0}+\cdots+k_{m-2}=n}\right) \frac{n !}{k_{0} ! \cdots k_{m-2} !} \prod_{\nu=0}^{m-2} x_{v}^{k_{\nu},}
\end{aligned}
$$

where the summation is taken over all $k_{0}, \ldots, k_{m-2} \in \mathbb{N}_{0}$ such that $k_{0}+\cdots+k_{m-2}=n$.

In addition, for $z \in \mathbb{C}$ and $\left|\lambda z^{\beta-\alpha}\right|<1$, we have

$$
\begin{aligned}
& \frac{z^{j-1-\beta-\sum_{\nu=0}^{m-2}\left(\beta-\alpha_{\nu}\right) k_{\nu}}}{\left(z^{\alpha-\beta}-\lambda\right)^{n+1}} \\
& =\frac{z^{(\alpha-\beta)-\left(\alpha+1-j+\sum_{\nu=0}^{m-2}\left(\beta-\alpha_{\nu}\right) k_{\nu}\right)}}{\left(z^{\alpha-\beta}-\lambda\right)^{n+1}} \\
& =\frac{1}{n !} \mathscr{L}_{\Delta, t_{0}}\left\{\frac{\partial^{n}}{\partial \lambda^{n}} F_{\nabla-\beta, \alpha+1-j+\sum_{\nu=0}^{m-2}\left(\beta-\alpha_{\nu}\right) k_{\nu}}\left(\lambda ; t, t_{0}\right)\right\}(z) .
\end{aligned}
$$

From (178), (180), and (182), we derive the solution to (176), as

$$
y(t)=\sum_{j=1}^{l} d_{j} y_{j}(t),
$$

which shows that arbitrary solution $y(t)$ can be expressed by $y_{j}(t), j=1, \ldots, l$, where $y_{j}(t)(j=1, \ldots, l)$ are given by $(177)$. For $q, j=1, \ldots, l$, the direct evaluation yields

$$
\begin{aligned}
& D_{\Delta, t_{0}}^{\alpha-q} y_{j}(t) \\
&=D_{\Delta, t_{0}}^{\alpha-q}\left\{\sum_{n=0}^{\infty}\left(\sum_{k_{0}+\cdots+k_{m-2}=n}\right) \frac{1}{k_{0} ! \cdots k_{m-2} !}\left[\prod_{\nu=0}^{m-2}\left(A_{\nu}\right)^{k_{\nu}}\right]\right. \\
&\left.\quad \times \frac{\partial^{n}}{\partial \lambda^{n}} F_{\Delta} F_{\alpha-\beta, \alpha+1-j+\sum_{\nu=0}^{m-2}\left(\beta-\alpha_{\nu}\right) k_{\nu}}\left(\lambda ; t, t_{0}\right)\right\} \\
&=\sum_{n=0}^{\infty}\left(\sum_{k_{0}+\cdots+k_{m-2}=n}\right) \frac{1}{k_{0} ! \cdots k_{m-2} !}\left[\prod_{\nu=0}^{m-2}\left(A_{\nu}\right)^{k_{\nu}}\right] \\
& \quad \times \frac{\partial^{n}}{\partial \lambda^{n}} D_{\Delta, t_{0}}^{\alpha-q}\left[\sum_{s=0}^{\infty} \lambda^{s} \widehat{h}_{s(\alpha-\beta)+\alpha-j+\sum_{\nu=0}^{m-2}\left(\beta-\alpha_{\nu}\right) k_{\nu}}\left(t, t_{0}\right)\right] \\
&=\sum_{n=0}^{\infty}\left(\sum_{k_{0}+\cdots+k_{m-2}=n}\right) \frac{1}{k_{0} ! \cdots k_{m-2} !}\left[\prod_{\nu=0}^{m-2}\left(A_{\nu}\right)^{k_{\nu}}\right] \\
& \quad \times \frac{\partial^{n}}{\partial \lambda^{n}} \sum_{s=0}^{\infty} \lambda^{s} \widehat{h}_{s(\alpha-\beta)+\sum_{\nu=0}^{m-2}\left(\beta-\alpha_{\nu}\right) k_{\nu}+q-j}\left(t, t_{0}\right) .
\end{aligned}
$$

For $q>j, D_{\Delta, t_{0}}^{\alpha-q} y_{j}\left(t_{0}\right)=0$, and for $q=j, D_{\Delta, t_{0}}^{\alpha-q} y_{j}\left(t_{0}\right)=$ 1. Thus we have $W_{\alpha}\left(t_{0}\right)=1$. It follows from Lemma 58 that the functions $y_{j}(t), j=1,2, \ldots, l$ in (177) are linearly independent solutions and then they yield the fundamental system of solutions to (176). Furthermore, if $q<j$, then we rewrite (184) as follows:

$$
\begin{aligned}
D_{\Delta, t_{0}}^{\alpha-q} y_{j} & (t) \\
= & D_{\Delta, t_{0}}^{\alpha-q} \widehat{h}_{\alpha-j}\left(t, t_{0}\right) \\
& +\sum_{s=1}^{\infty} \lambda^{s} \widehat{h}_{s(\alpha-\beta)+\sum_{v=0}^{m-2}\left(\beta-\alpha_{\nu}\right) k_{\nu}+q-j}\left(t, t_{0}\right) \\
& +\sum_{n=1}^{\infty}\left(\sum_{k_{0}+\cdots+k_{m-2}=n}\right) \frac{1}{k_{0} ! \cdots k_{m-2} !} \\
& \times\left[\prod_{\nu=0}^{m-2}\left(A_{v}\right)^{k_{\nu}}\right] \frac{\partial^{n}}{\partial \lambda^{n}} \widehat{h}_{\sum_{v=0}^{m-2}\left(\beta-\alpha_{\nu}\right) k_{\nu}+q-j}\left(t, t_{0}\right) \\
& +\sum_{n=1}^{\infty}\left(\sum_{k_{0}+\cdots+k_{m-2}=n}\right) \frac{1}{k_{0} ! \cdots k_{m-2} !}
\end{aligned}
$$




$$
\begin{aligned}
& \times\left[\prod_{\nu=0}^{m-2}\left(A_{\nu}\right)^{k_{\nu}}\right] \frac{\partial^{n}}{\partial \lambda^{n}} \sum_{s=1}^{\infty} \lambda^{s} \widehat{h}_{s(\alpha-\beta)+\sum_{v=0}^{m-2}\left(\beta-\alpha_{\nu}\right) k_{v}+q-j}\left(t, t_{0}\right) \\
= & \sum_{s=1}^{\infty} \lambda^{s} \widehat{h}_{s(\alpha-\beta)+q-j}\left(t, t_{0}\right) \\
& +\sum_{n=1}^{\infty}\left(\sum_{k_{0}+\cdots+k_{m-2}=n}\right) \frac{1}{k_{0} ! \cdots k_{m-2} !} \\
& \times\left[\prod_{\nu=0}^{m-2}\left(A_{\nu}\right)^{k_{\nu}}\right] \frac{\partial^{n}}{\partial \lambda^{n}} \widehat{h}_{\sum_{v=0}^{m-2}\left(\beta-\alpha_{\nu}\right) k_{\nu}+q-j}\left(t, t_{0}\right) \\
& +\sum_{n=1}^{\infty}\left(\sum_{k_{0}+\cdots+k_{m-2}=n}\right) \frac{1}{k_{0} ! \cdots k_{m-2} !} \\
& \times\left[\prod_{\nu=0}^{m-2}\left(A_{\nu}\right)^{k_{\nu}}\right] \frac{\partial^{n}}{\partial \lambda^{n}} \sum_{s=1}^{\infty} \lambda^{s} \widehat{h}_{s(\alpha-\beta)+\sum_{\nu=0}^{m-2}\left(\beta-\alpha_{\nu}\right) k_{\nu}+q-j}\left(t, t_{0}\right) .
\end{aligned}
$$

If $\alpha+1-l>\beta>\alpha_{m-2}+l-1$, then $s(\alpha-\beta)+q-j \geq$ $(\alpha-\beta)+1-l>0$ for $n=0, q, j=1, \ldots, l, s \in \mathbb{N}^{+}$, and $\sum_{\nu=0}^{m-2}\left(\beta-\alpha_{v}\right) k_{v}+q-j \geq \beta-\alpha_{i}+1-l \geq \beta-\alpha_{m-2}+1-l>$ 0 for $s=0, q, j=1, \ldots, l, n \in \mathbb{N}^{+}$. Besides, we also have $s(\alpha-\beta)+\sum_{\nu=0}^{m-2}\left(\beta-\alpha_{v}\right) k_{v}+q-j \geq(\alpha-\beta)+\beta-\alpha_{i}+1-l \geq$ $(\alpha-\beta)+\beta-\alpha_{m-2}+1-l>0$ for $q, j=1, \ldots, l, s, n \in \mathbb{N}^{+}$. These imply that $D_{\Delta, t_{0}}^{\alpha-q} y_{j}\left(t_{0}\right)=0$. Thus the relations in (151) are valid. The result follows.

\section{Nonhomogeneous Equations with Constant Coefficients}

In Section 7, we have applied the Laplace transform method to derive explicit solutions to the homogeneous equations (140) with the Liouville fractional derivatives. Here we use this approach to find particular solutions to the corresponding nonhomogeneous equations

$$
\begin{aligned}
& \sum_{k=1}^{m} A_{k} D_{\Delta, t_{0}}^{\alpha_{k}} y(t)+A_{0} y(t) \\
& \quad=f(t) \quad\left(t>t_{0} ; 0<\alpha_{1}<\cdots<\alpha_{m} ; m \in \mathbb{N}\right)
\end{aligned}
$$

with real $A_{k} \in \mathbb{R}(k=0, \ldots, m)$.

By (141)-(142), for suitable functions $y$, the Laplace transform of $D_{\Delta, t_{0}}^{\alpha} y$ is given by

$$
\mathscr{L}\left\{D_{\Delta, t_{0}}^{\alpha} y(t)\right\}\left(z, t_{0}\right)=z^{\alpha} \mathscr{L}\{y(t)\}\left(z, t_{0}\right)
$$

Applying the Laplace transform to (186) and taking (187) into account, we have

$$
\left[A_{0}+\sum_{k=1}^{m} A_{k} z^{\alpha_{k}}\right] \mathscr{L}\{y(t)\}\left(z, t_{0}\right)=\mathscr{L}\{f(t)\}\left(z, t_{0}\right) .
$$

Using the inverse Laplace transform $\mathscr{L}_{\Delta}^{-1}$ from here, we obtain a particular solution to (186) in the form

$$
y(t)=\mathscr{L}^{-1}\left[\frac{\mathscr{L}\{f(t)\}\left(z, t_{0}\right)}{A_{0}+\sum_{k=1}^{m} A_{k} z^{\alpha_{k}}}\right](t) .
$$

Using the Laplace convolution formula

$$
\mathscr{L}\{f * g\}\left(z, t_{0}\right)=\mathscr{L}\{f\}\left(z, t_{0}\right) \mathscr{L}\{g\}\left(z, t_{0}\right),
$$

we can introduce the Laplace fractional analog of the Green function as follows:

$$
\begin{gathered}
G_{\alpha_{1}, \ldots, \alpha_{m}}(t)=\mathscr{L}^{-1}\left\{\frac{1}{P_{\alpha_{1}, \ldots, \alpha_{m}}(z)}\right\}(t), \\
P_{\alpha_{1}, \ldots, \alpha_{m}}(z)=A_{0}+\sum_{k=1}^{m} A_{k} z^{\alpha_{k}}
\end{gathered}
$$

and we can express a particular solution of (152) in the form of the Laplace convolution $G_{\alpha_{1}, \ldots, \alpha_{m}}(t)$ and $h_{r}\left(t, t_{0}\right)$ :

$$
y(t)=\left(f * G_{\alpha_{1}, \ldots, \alpha_{m}}\right)(t) .
$$

Generally speaking, we can consider (186) with $A_{m}=1$. First we derive a particular solution to (186) with $m=1$ in the form

$$
D_{\Delta, t_{0}}^{\alpha} y(t)-\lambda y(t)=f(t) \quad\left(t>t_{0} ; \alpha>0\right) .
$$

Theorem 64. Let $\alpha>0, \lambda \in \mathbb{R}$. Then (193) is solvable, and its particular solution has the form

$$
y(t)=\left(f *{ }_{\Delta} F_{\alpha, \alpha}\left(\lambda, s, t_{0}\right)\right)(t)
$$

provided that the integral in the right-hand side of (194) is convergent.

Proof. Equation (193) is the same as (186) with $m=1, \alpha_{1}=\alpha$, and $A_{1}=1, A_{0}=-\lambda$ and (191) takes the form

$$
G_{\alpha}(t)=\mathscr{L}^{-1}\left\{\frac{1}{z^{\alpha}-\lambda}\right\}(t)={ }_{\Delta} F_{\alpha, \alpha}\left(\lambda, t, t_{0}\right) .
$$

Thus (192), with $G_{\alpha_{1}, \ldots, \alpha_{m}}(t)=G_{\alpha}(t)$, yields (194). Theorem is proved.

Next we derive a particular solution to (186) with $m=2$ of the form

$$
\begin{array}{r}
D_{\Delta, t_{0}}^{\alpha} y(t)-\lambda D_{\Delta, t_{0}}^{\beta} y(t)-\mu y(t)=f(t), \\
\left(t>t_{0} ; \alpha>\beta>0\right) .
\end{array}
$$

Theorem 65. Let $\alpha>\beta>0, \lambda, \mu \in \mathbb{R}$. Then (196) is solvable, and its particular solution has the form

$$
\begin{gathered}
y(t)=\left(f * G_{\alpha, \beta ; \lambda, \mu}(s)\right)(t), \\
G_{\alpha, \beta ; \lambda, \mu}(t)=\sum_{k=0}^{\infty} \frac{\mu^{k}}{k !} \frac{\partial^{k}}{\partial \lambda^{k}} \Delta F_{\alpha-\beta, \alpha+k \beta}\left(\lambda, t, t_{0}\right)
\end{gathered}
$$

provided that the series in (198) and the integral in (197) are convergent. 
Proof. Equation (196) is the same as (186) with $m=2, \alpha_{2}=\alpha$, $\alpha_{1}=\beta, A_{2}=1, A_{1}=-\lambda$, and $A_{0}=-\mu$, and (191) is given by

$$
G_{\alpha, \beta}(t)=\mathscr{L}^{-1}\left\{\frac{1}{z^{\alpha}-\lambda z^{\beta}-\mu}\right\}(t) .
$$

According to (168) for $z \in \mathbb{C}$ and $\left|\mu z^{-\beta} /\left(z^{\alpha-\beta}-\lambda\right)\right|<1$, we have

$$
G_{\alpha, \beta ; \lambda, \mu}(t)=\mathscr{L}^{-1}\left\{\sum_{n=0}^{\infty} \mu^{n} \frac{z^{-\beta-n \beta}}{\left(z^{\alpha-\beta}-\lambda\right)^{n+1}}\right\}(t) .
$$

In addition, for $z \in \mathbb{C}$ and $\left|\lambda z^{\beta-\alpha}\right|<1$, we have

$$
\frac{z^{-\beta-n \beta}}{\left(z^{\alpha-\beta}-\lambda\right)^{n+1}}=\frac{1}{n !} \mathscr{L}\left\{\frac{\partial^{n}}{\partial \lambda^{n}}{ }_{\Delta} F_{\alpha-\beta, \alpha+n \beta}\left(\lambda, t, t_{0}\right)\right\}\left(z, t_{0}\right)
$$

and hence (200) takes the following form:

$$
G_{\alpha, \beta ; \lambda, \mu}(t)=\sum_{n=0}^{\infty} \frac{\mu^{n}}{n !} \frac{\partial^{n}}{\partial \lambda^{n}}{ }_{\Delta} F_{\alpha-\beta, \alpha+n \beta}\left(\lambda, t, t_{0}\right)
$$

Thus the result in (197) follows from (192) with $G_{\alpha_{1}, \ldots, \alpha_{m}}(t)=$ $G_{\alpha, \beta ; \lambda, \mu}(t)$.

Finally, we find a particular solution to (186) with any $m \in$ $\mathbb{N} \backslash\{1,2\}$. It is convenient to rewrite (186) just as (176) in the form

$$
\begin{array}{r}
D_{\Delta, t_{0}}^{\alpha} y(t)-\lambda D_{\Delta, t_{0}}^{\beta} y(t)-\sum_{k=1}^{m-2} A_{k} D_{\Delta, t_{0}}^{\alpha_{k}} y(t)-A_{0} y(t) \\
=\widehat{h}_{r}\left(t, t_{0}\right) \quad\left(t>t_{0}\right)
\end{array}
$$

with $m \in \mathbb{N} \backslash\{1,2\}, 0<\alpha_{1}<\cdots<\alpha_{m-2}<\beta<\alpha$, and $\lambda, A_{0}, \ldots, A_{m-2} \in \mathbb{R}$.

Theorem 66. Let $m \in \mathbb{N} \backslash\{1,2\}, 0=\alpha_{0}<\alpha_{1}<\cdots<\alpha_{m-2}<$ $\beta<\alpha$, and let $\lambda, A_{0}, \ldots, A_{m-2} \in \mathbb{R}$. Then (203) is solvable, and its particular solution has the form

$$
\begin{gathered}
y(t)=\left(f * G_{\alpha_{1}, \ldots, \alpha_{m-2}, \beta, \alpha ; \lambda}(s)\right)(t) \\
G_{\alpha_{1}, \ldots, \alpha_{m-2}, \beta, \alpha ; \lambda}(t) \\
=\sum_{n=0}^{\infty}\left(\sum_{k_{0}+\cdots+k_{m-2}=n}\right) \frac{1}{k_{0} ! \cdots k_{m-2} !} \\
\times\left[\prod_{v=0}^{m-2}\left(A_{v}\right)^{k_{v}}\right] \frac{\partial^{n}}{\partial \lambda^{n}} F_{\alpha-\beta, \alpha+\sum_{v=0}^{m-2}\left(\beta-\alpha_{v}\right) k_{v}}\left(\lambda, t, t_{0}\right)
\end{gathered}
$$

provided that the series (205) and integral in (204) are convergent. The inner sum is taken over all $k_{0}, \ldots, k_{m-2}$ such that $k_{0}+\cdots+k_{m-2}=n$.
Proof. Equation (203) is the same equation as (186) with $\alpha_{m}=$ $\alpha, \alpha_{m-1}=\beta, A_{m}=1, \ldots, A_{m-1}=-\lambda$, and with $-A_{k}$ instead of $A_{k}$ for $k=0, \ldots, m-2$. Since $\alpha_{0}=0$, (191) takes the form

$$
G_{\alpha_{1}, \ldots, \alpha_{m-2}, \beta, \alpha ; \lambda}(t)=\mathscr{L}^{-1}\left\{\frac{1}{z^{\alpha}-\lambda \alpha^{\beta}-\sum_{k=0}^{m-2} A_{k} z^{\alpha_{k}}}\right\}(t) .
$$

For $z \in \mathbb{C}$ and $\left|\sum_{k=0}^{m-2} A_{k} z^{\alpha_{k}-\beta} /\left(z^{\alpha-\beta}-\lambda\right)\right|<1$, in accordance with (180), we have

$$
\begin{aligned}
G_{\alpha_{1}, \ldots, \alpha_{m-2}, \beta, \alpha ; \lambda} & (t) \\
=\mathscr{L}^{-1} & \left\{\sum_{n=0}^{\infty}\left(\sum_{k_{0}+\cdots+k_{m-2}=n}\right) \frac{n !}{k_{0} ! \cdots k_{m-2} !}\right. \\
& \left.\times\left[\prod_{v=0}^{m-2}\left(A_{v}\right)^{k_{v}}\right] \frac{z^{-\beta-\sum_{v=0}^{m-2}\left(\beta-\alpha_{v}\right) k_{v}}}{\left(z^{\alpha-\beta}-\lambda\right)^{n+1}}\right\}(t) .
\end{aligned}
$$

For $z \in \mathbb{C}$ and $\left|\lambda z^{\beta-\alpha}\right|<1$, we have

$$
\begin{aligned}
& \frac{z^{-\beta-\sum_{v=0}^{m-2}\left(\beta-\alpha_{v}\right) k_{v}}}{\left(z^{\alpha-\beta}-\lambda\right)^{n+1}} \\
& \quad=\frac{1}{n !} \mathscr{L}\left\{\frac{\partial^{n}}{\partial \lambda^{n}} \Delta F_{\alpha-\beta, \alpha+\sum_{v=0}^{m-2}\left(\beta-\alpha_{v}\right) k_{v}}\left(\lambda, t, t_{0}\right)\right\}\left(z, t_{0}\right) .
\end{aligned}
$$

The proof is finished.

As in the case of ordinary differential equations, a general solution to the nonhomogeneous equation (186) is a sum of a particular solution to this equation and of the general solution to the corresponding homogeneous equation (140). Therefore, the results established in Section 7 and in Section 8 can be used to derive general solutions to the nonhomogeneous equations (193), (196), and (203). The following statements can thus be derived from Theorems 59, 64, 61, 65 and Theorems 63 and 66, respectively.

Theorem 67. Let $l-1<\alpha \leq l(l \in \mathbb{N}), \lambda \in \mathbb{R}$. Then (193) is solvable, and its general solution is given by

$$
y(t)=\sum_{j=1}^{l} c_{j \Delta} F_{\alpha, \alpha+1-j}\left(\lambda, t, t_{0}\right)+f(t)
$$

where $c_{j}(j=1, \ldots, l)$ are arbitrary real constants.

Theorem 68. Let $l-1<\alpha \leq l(l \in \mathbb{N}), 0<\beta<\alpha, \lambda, \mu \in \mathbb{R}$. Then (196) is solvable, and its general solution has the form

$$
\begin{aligned}
y(t)= & \sum_{j=1}^{l} c_{j} \sum_{n=0}^{\infty} \frac{\mu^{n}}{n !} \frac{\partial^{n}}{\partial \lambda^{n}}{ }_{\Delta} F_{\alpha-\beta, \alpha+n \beta+1-j}\left(\lambda, t, t_{0}\right) \\
& +\left(f * G_{\alpha, \beta, \lambda, \mu}(s)\right)(t),
\end{aligned}
$$

where $G_{\alpha, \beta, \lambda, \mu}(t)$ is given by (198) and $c_{j}(j=1, \ldots, l)$ are arbitrary real constants. 
Theorem 69. Let $m \in \mathbb{N} \backslash\{1,2\}, l-1<\alpha \leq l(l \in \mathbb{N})$, let $\beta$ and $\alpha_{1}, \alpha_{2}, \ldots, \alpha_{m-2}$ be such that $\alpha>\beta>\alpha_{m-2}>\cdots>\alpha_{1}>$ $\alpha_{0}=0$ and $\alpha-l+1 \geq \beta$, and let $\lambda, A_{0}, \ldots, A_{m-2} \in \mathbb{R}$. Then (203) is solvable, and its general solution is given by

$$
\begin{aligned}
y(t)= & \sum_{j=1}^{l} c_{j} \sum_{n=0}^{\infty}\left(\sum_{k_{0}+\cdots+k_{m-2}=r}\right) \frac{1}{k_{0} ! \cdots k_{m-2} !} \\
& \times\left[\prod_{v=0}^{m-2}\left(A_{v}\right)^{k_{v}}\right] \\
& \cdot \frac{\partial^{n}}{\partial \lambda^{n}} F_{\alpha-\beta, \alpha+1-j+\sum_{v=0}^{m-2}\left(\beta-\alpha_{v}\right) k_{v}}\left(\lambda, t, t_{0}\right) \\
& +\left(f * G_{\alpha_{1}, \ldots, \alpha_{m-2, \beta, \alpha ; \lambda}}(s)\right)(t),
\end{aligned}
$$

where $G_{\alpha_{1}, \ldots, \alpha_{m-2, \beta, \alpha ; \lambda}}(t)$ is given by (205) and $c_{j}(j=1, \ldots, l)$ are arbitrary real constant.

\section{Conclusions}

In this paper, we first give a generalized definition of fractional $\Delta$-power function on general time scale by inversion of Laplace transform and shift transform. The fractional $\Delta$ power function on a time scale is an important basis of fractional $\Delta$-integral and fractional $\Delta$-differential on time scales. Then, based on the fractional $\Delta$-power function, we give a new definition of Riemann-Liouville fractional $\Delta$-integral and Riemann-Liouville fractional $\Delta$-derivative on time scales. Some of properties of Riemann-Liouville fractional $\Delta$-integral and Riemann-Liouville fractional $\Delta$ derivative on time scales are studied in detail. On this basis, equivalencies of Cauchy type problem with RiemannLiouville fractional $\Delta$-derivative and nonlinear Volterra integral equation are obtained. By employing Laplace transform, we derive explicit solutions to homogeneous and nonhomogeneous equations of Riemann-Liouville fractional $\Delta$ derivative with constant coefficient. We give the conditions when the solutions of linear fractional differential equation will be linearly independent and when these linearly independent solutions form the fundamental system of solutions. On the other hand, we know that continuous fractional differential equation theory and discrete fractional difference equation theory have been studied by many authors, the existence and uniqueness of the solution to the boundary value problems for fractional differential equations have been studied a lot by many methods involving partial order method, fixed point method, lower and upper solutions method, transform method, and so on. For example, for the recent developments about continuous fractional differential equations and discrete fractional difference equations, one can refer to $[3,5-11]$ and the references therein. However, the fractional differential equation theory on time scales is still an open problem. Therefore, the authors believe that the present work will potentiate further research in the study of fractional differential equation theory on time scales in the future.

\section{Conflict of Interests}

The authors declare that there is no conflict of interests regarding the publication of this paper.

\section{Authors' Contribution}

All authors contributed equally and significantly to the writing of this paper. All authors read and approved the final paper.

\section{Acknowledgments}

First, the authors are very grateful to the referees for their careful reading of the paper, and lots of valuable comments and suggestions, which greatly improve this manuscript. Next, this work was supported by the National Natural Science Foundation of China (11171286) and by Jiangsu Province Colleges and Universities Graduate Scientific Research Innovative Program (CXZZ12-0974).

\section{References}

[1] M. Holm, "Sum and difference compositions in discrete fractional calculus," CUBO A Mathematical Journal, vol. 13, no. 3, pp. 153-184, 2011.

[2] F. M. Atici and P. W. Eloe, "A transform method in discrete fractional calculus," International Journal of Difference Equations, vol. 2, pp. 165-176, 2007.

[3] A. A. Kilbas, H. M. Srivastava, and J. J. Trujillo, Theory and Applications of Fractional Differentia Equation, Elsevier B.V., London, UK, 2006.

[4] J. Cheng, Theory of Fractional Difference Equation, Xiamen University Press, Fujian, China, 2011.

[5] C. Kou, H. Zhou, and Y. Yan, "Existence of solutions of initial value problems for nonlinear fractional differential equations on the half-axis," Nonlinear Analysis, Theory, Methods and Applications, vol. 74, no. 17, pp. 5975-5986, 2011.

[6] R. P. Agarwal, V. Lakshmikantham, and J. J. Nieto, "On the concept of solution for fractional differential equations with uncertainty," Nonlinear Analysis, Theory, Methods and Applications, vol. 72, no. 6, pp. 2859-2862, 2009.

[7] Y. Liu, W. Zhang, and X. Liu, "A sufficient condition for the existence of a positive solution for a nonlinear fractional differential equation with the Riemann-Liouville derivative," Applied Mathematics Letters, vol. 25, no. 11, pp. 1986-1992, 2012.

[8] V. Lakshmikantham and A. S. Vatsala, "Basic theory of fractional differential equations," Nonlinear Analysis, Theory, Methods and Applications, vol. 69, no. 8, pp. 2677-2682, 2008.

[9] K. Diethelm, The Analysis of Fractional Differential Equations, Springer, Berlin, Germany, 2010.

[10] R. A. C. Ferreira and D. M. Torres, "Fractional hdifference equations arising from the calculus of variations," http://arxiv.org/abs/1101.5904v1 .

[11] C. S. Goodrich, "Existence and uniqueness of solutions to a fractional difference equation with nonlocal conditions," Computers and Mathematics with Applications, vol. 61, no. 2, pp. 191-202, 2011.

[12] M. Bohner and A. Peterson, Dynamic Equations on Time Scales, An Introduction with Applications, Birkhäauser, Boston, Mass, USA, 2001. 
[13] M. Bohner and A. Peterson, Advances in Dynamic Equations on Time Scales, Birkhäauser, Boston, Mass, USA, 2003.

[14] M. Bohner and G. S. Guseinov, "Partial differentiation on time scales," Dynamic Systems and Applications, vol. 13, no. 3-4, pp. 351-379, 2004.

[15] J. M. Davis, I. A. Gravagne, B. J. Jackson, R. J. Marks II, and A. A. Ramos, "The Laplace transform on time scales revisited," Journal of Mathematical Analysis and Applications, vol. 332, no. 2, pp. 1291-1307, 2007.

[16] P. A. Williams, Unifying fractional calculus with time scales [Ph.D. thesis], The University of Melbourne, Parkville, Australia, 2012.

[17] N. R. D. O. Bastos, Fractional calculus on time scales [Ph.D. thesis], The University of Aveiro, Aveiro, Portugal, 2012.

[18] M. Bohner and G. S. Guseinov, "The convolution on time scales," Abstract and Applied Analysis, vol. 2007, Article ID 58373, 24 pages, 2007. 


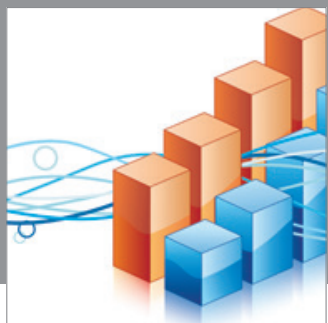

Advances in

Operations Research

mansans

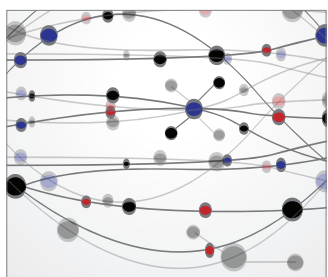

The Scientific World Journal
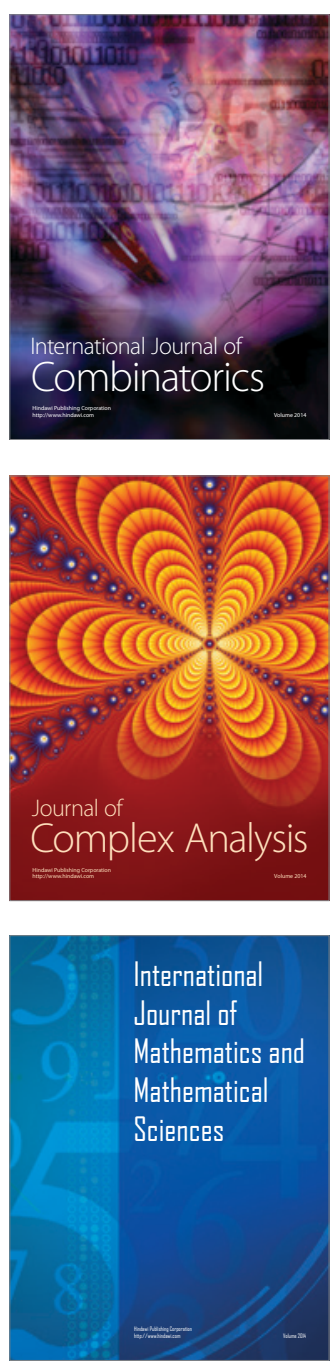
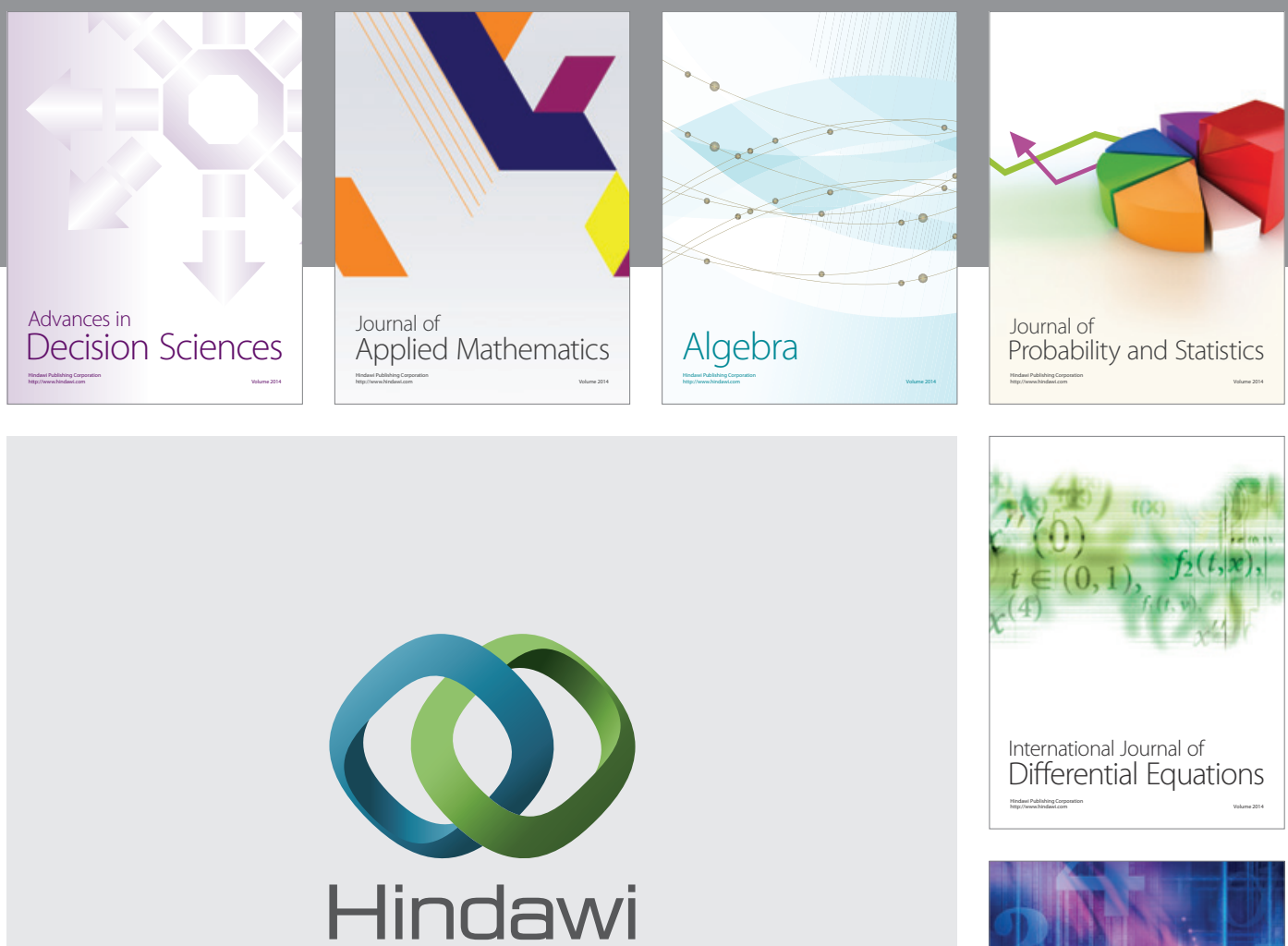

Submit your manuscripts at http://www.hindawi.com
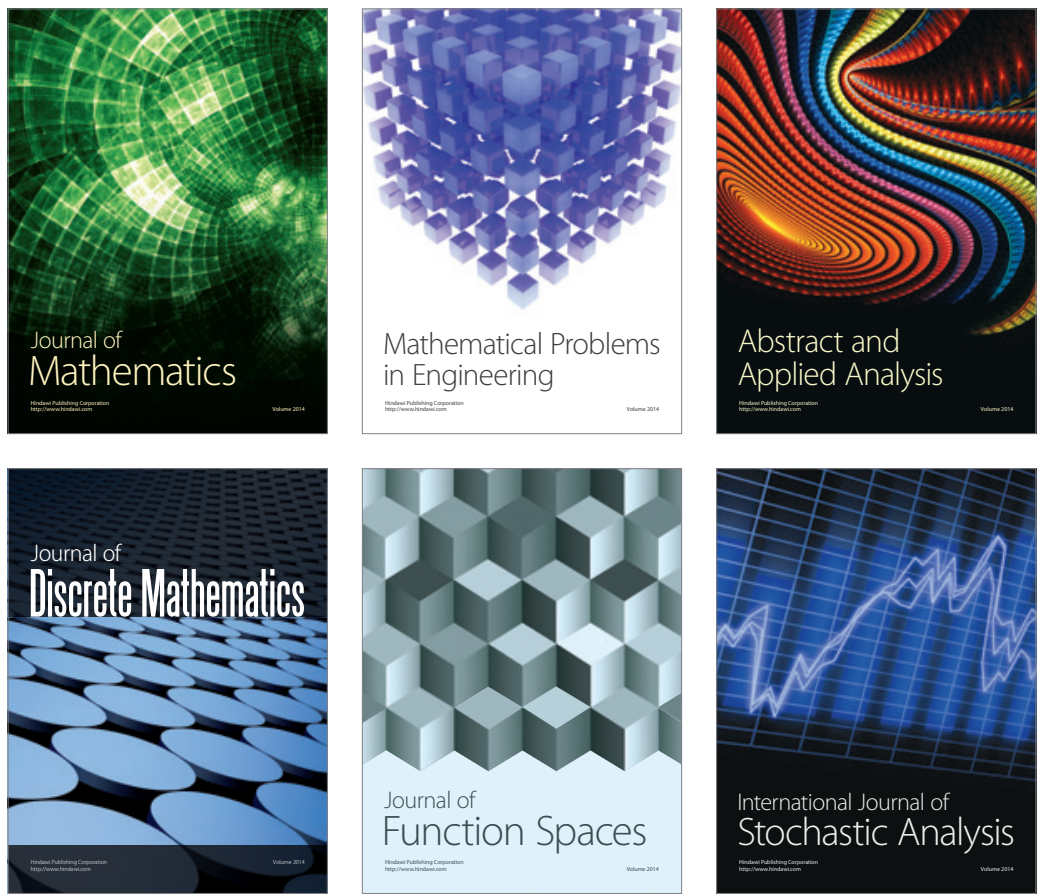

Journal of

Function Spaces

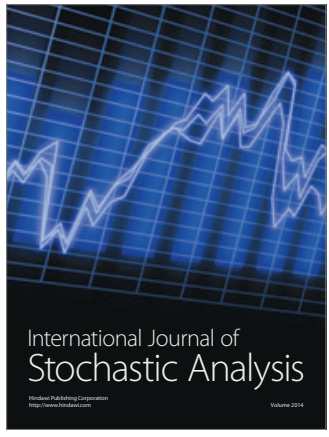

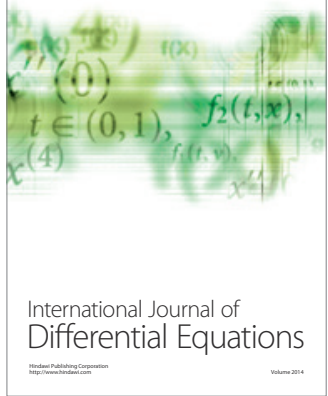
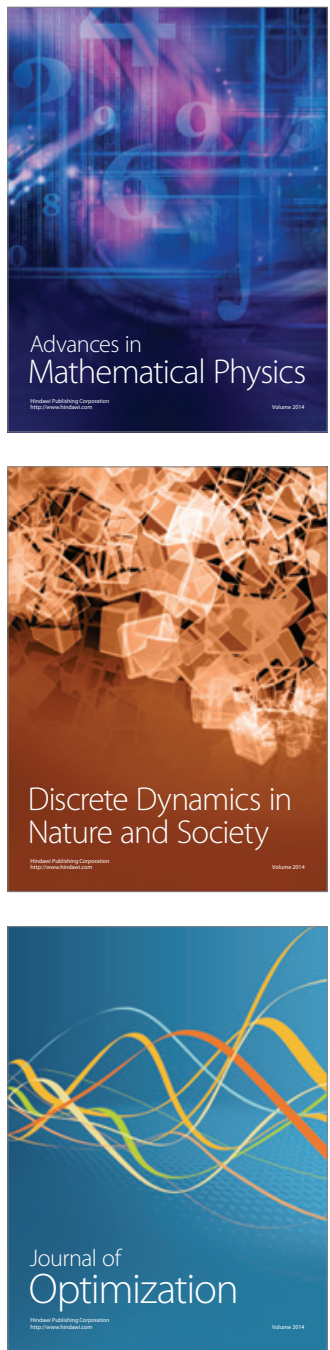